\title{
First-Order Adaptive Azimuthal Null-Steering for the Suppression of Two Directional Interferers
}

\author{
René M. M. Derkx \\ Digital Signal Processing Group, High Tech Campus 36, 5656 AE Eindhoven, The Netherlands \\ Correspondence should be addressed to René M. M. Derkx, renederkx@online.nl
}

Received 21 July 2009; Revised 10 November 2009; Accepted 15 December 2009

Academic Editor: Simon Doclo

Copyright () 2010 René M. M. Derkx. This is an open access article distributed under the Creative Commons Attribution License, which permits unrestricted use, distribution, and reproduction in any medium, provided the original work is properly cited.

\begin{abstract}
An azimuth steerable first-order superdirectional microphone response can be constructed by a linear combination of three eigenbeams: a monopole and two orthogonal dipoles. Although the response of a (rotation symmetric) first-order response can only exhibit a single null, we will look at a slice through this beampattern lying in the azimuthal plane. In this way, we can define maximally two nulls in the azimuthal plane which are symmetric with respect to the main-lobe axis. By placing these two nulls on maximally two directional sources to be rejected and compensating for the drop in level for the desired direction, we can effectively reject these directional sources without attenuating the desired source. We present an adaptive null-steering scheme for adjusting the beampattern so as to obtain this suppression of the two directional interferers automatically. Closed-form expressions for this optimal null-steering are derived, enabling the computation of the azimuthal angles of the interferers. It is shown that the proposed technique has a good directivity index when the angular difference between the desired source and each directional interferer is at least 90 degrees.
\end{abstract}

\section{Introduction}

In applications such as hands-free communication and voice control systems, the microphone signal does not only contain the desired sound-source (e.g., a speech signal) but can also contain undesired directional interferers and background noise (e.g., diffuse-noise). To reduce the amount of noise and minimize the influence of interferers, we can use a microphone array and apply beamforming techniques to steer the main-lobe of a beam towards the desired sourcesignal, for example, a speech signal. In this paper, we focus on arrays where the wavelength of the sound is much large than the size of the array. These arrays are therefore called "Small Microphone Arrays." When using omnidirectional (monopole) microphones in a small microphone array configuration, additive beamformers like delay-and-sum are not able to obtain a sufficient directivity as the beamwidth deteriorates for larger wavelengths $[1,2]$. A common method to obtain improved directivity is to apply superdirective beamforming techniques. In this paper, we will focus on first-order superdirective beamforming. (The term "firstorder" is used to indicate that the directivity-pattern of the superdirectional response is constructed by means of a linear combination of a pressure and velocity (first-order spatial derivative of the pressure field) response.)

A first method to obtain this first-order superdirectivity is by using microphone-arrays with omnidirectional microphone elements and to apply beamforming-techniques with asymmetrical filter-coefficients [3]. Basically, this asymmetrical filtering corresponds to subtraction of signals, like in delay-and-subtract techniques $[4,5]$ or by taking spatial derivatives of the sound pressure field $[6,7]$. As subtraction leads to smaller signals for low frequencies, a first-order integrator needs to be applied to equalize the frequency-response, resulting in an increased sensitivity ( $20 \mathrm{~dB} /$ decade) for sensor-noise and increased sensitivity for mismatches in microphones characteristics $[8,9]$ for the lower-frequency-range.

A second method to obtain first-order superdirectivity is by using microphone-arrays with first-order unidirectional microphone elements. As the separate uni-directional microphone elements already have a first-order superdirective response, consisting out of a sum of a pressure and a velocity response, the beamformer can simply be constructed 

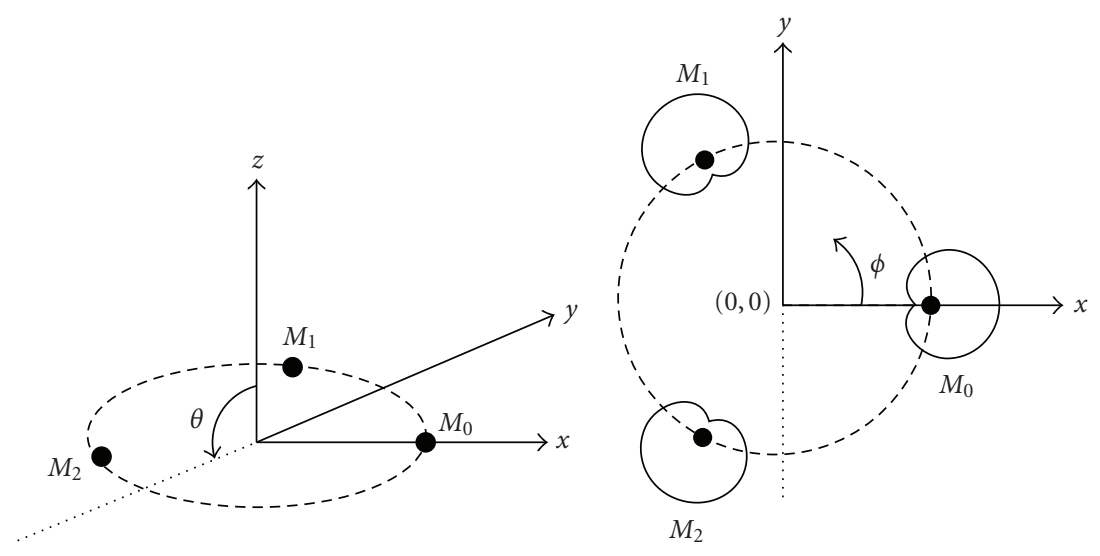

FIgURE 1: Circular array geometry with three cardioid microphones.

by a linear combination of the uni-directional microphone signals. In such an approach, there is no need to apply a firstorder integrator (as was the case for omni-directional microphone elements), and we avoid a $20 \mathrm{~dB} /$ decade increased sensitivity for sensor-noise [7]. Nevertheless, uni-directional microphones may have a low-frequency roll-off, which can be compensated for by means of proper equalization techniques. Throughout this paper, we will assume that the uni-directional microphones have a flat frequency response.

We focus on the construction of first-order superdirectional beampatterns where the nulls of the beampattern are steered to the directional interferers, while having a unity response in the direction of the desired sound-source. In Section 2, we construct a monopole and two orthogonal dipole responses (known as "eigen-beams" [10,11]) out of a circular array of three first-order cardioid microphone elements $M_{0}, M_{1}$, and $M_{2}$ (with a heart-shaped directional pattern), as shown in Figure 1. Here $\theta$ and $\phi$ are the standard spherical coordinate angles: elevation and azimuth.

Based on these eigenbeams, we are able to construct arbitrary first-order responses that can be steered with the main-lobe in any azimuthal direction (see Section 2). Although the (rotation symmetric) first-order response can only exhibit a single null, we will look at a slice through the beampattern lying in the azimuthal plane. In this way, we can define maximally two nulls in the azimuthal plane which are symmetric with respect to the main-lobe axis. By placing these two nulls on the two directional sources to be rejected and compensating for the drop in level for the desired direction, we can effectively reject the directional sources without attenuating the desired source. In Section 3 expressions are derived for this beampattern synthesis.

To develop an adaptive null-steering algorithm, we first show in Section 4 how the superdirective beampattern can be synthesized via the Generalized Sidelobe Canceller (GSC) [12]. This GSC enables us to optimize a cost-function in an unconstrained manner with a gradient descent searchmethod that is described in Section 5. Furthermore, the GSC enables tracking of the angles of the separate directional interferers, which is validated by means of simulations and experiments in Section 6. Finally, in Section 7, conclusions are given.

\section{Construction of Eigenbeams}

We know from $[7,9]$ that by using a circular array of at least three (omni- or uni-directional microphone) sensors in a planar geometry and applying signal processing techniques, it is possible to construct a first-order superdirectional response. This superdirectional response can be steered with its main-lobe to any desired azimuthal angle and can be adjusted to have any first-order directivity pattern. As mentioned in the introduction, we will use three unidirectional cardioid microphones (with a heart-shaped directional pattern) in a circular configuration, where the main-lobes of the three cardioid responses are pointed outwards, as shown in Figure 1.

The responses of the three cardioid microphones $M_{0}, M_{1}$, and $M_{2}$ are given by, respectively, $E_{c}^{0}(r, \theta, \phi), E_{c}^{1}(r, \theta, \phi)$, and $E_{c}^{2}(r, \theta, \phi)$, having their main-lobes at, respectively, $\phi=0$, $2 \pi / 3$, and $4 \pi / 3$ radians. Assuming that we have no sensornoise, the $n$th cardioid microphone response, with $n=$ $0,1,2$, for a harmonic plane-wave with frequency $f$ is ideally given by [11]

$$
E_{c}^{n}(r, \theta, \phi)=A_{n} e^{j \psi_{n}}
$$

The magnitude-response $A_{n}$ and phase-response $\psi_{n}$ of the $n$th cardioid microphone are given by, respectively:

$$
\begin{gathered}
A_{n}=\frac{1}{2}+\frac{1}{2} \cos \left(\phi-\frac{2 n \pi}{3}\right) \sin \theta, \\
\psi_{n}=\frac{2 \pi f}{c} \sin \theta\left(x_{n} \cos \phi+y_{n} \sin \phi\right) .
\end{gathered}
$$

Here $c$ is the speed of sound and $x_{n}$ and $y_{n}$ are the $x$ and $y$ coordinates of the $n$th microphone (as shown in Figure 1), given by

$$
\begin{aligned}
& x_{n}=r \cos \left(\phi-\frac{2 n \pi}{3}\right), \\
& y_{n}=r \sin \left(\phi-\frac{2 n \pi}{3}\right),
\end{aligned}
$$




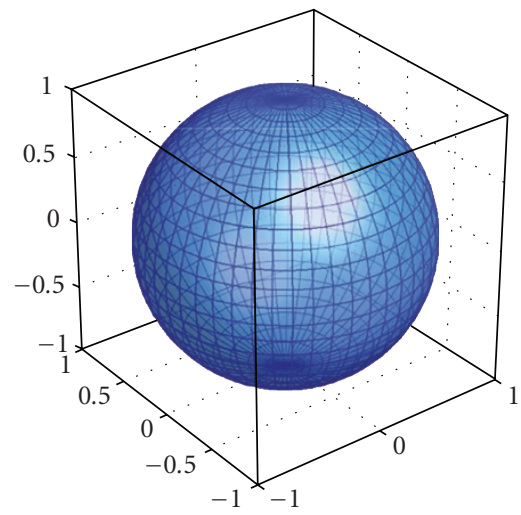

(a) $E_{m}(\theta, \phi)$

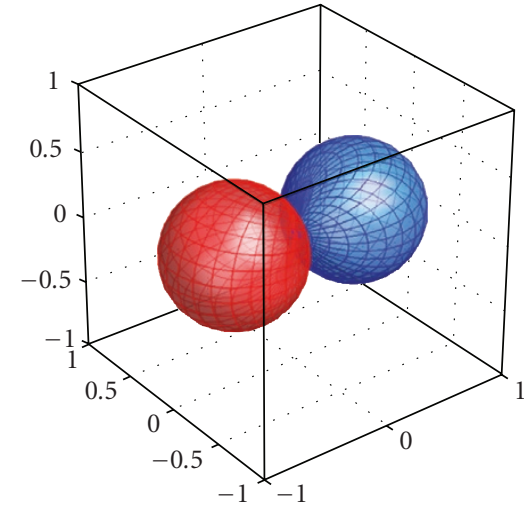

(b) $E_{d}^{0}(\theta, \phi)$

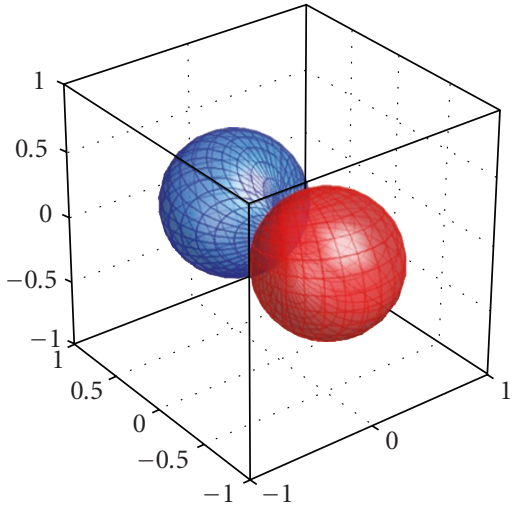

(c) $E_{d}^{\pi / 2}(\theta, \phi)$

FIgURE 2: Eigenbeams (monopole and two orthogonal dipoles).

with $r$ being the radius of the circle on which the microphones are located.

We can simplify (3) as

$$
\psi_{n}=\frac{2 \pi f}{c} r \sin \theta \cos \left(\frac{2 n \pi}{3}\right) .
$$

From the three cardioid microphone responses, we can construct the circular harmonics [7], also known as "eigenbeams" [10, 11]), by using the 3-point Discrete Fourier Transform (DFT) with the three microphones as inputs. This DFT produces three phase-modes $P_{i}(r, \theta, \phi)$ [7] with $i=$ $1,2,3$ :

$$
\begin{aligned}
P_{0}(r, \theta, \phi) & =\frac{1}{3} \sum_{n=0}^{2} E_{c}^{n}(r, \theta, \phi), \\
P_{1}(r, \theta, \phi) & =\left[P_{2}(r, \theta, \phi)\right]^{*} \\
& =\frac{1}{3} \sum_{n=0}^{2} E_{c}^{n}(r, \theta, \phi) e^{-j 2 \pi n / 3},
\end{aligned}
$$

with $j=\sqrt{-1}$ and $*$ being the complex-conjugate operator.

Via the phase-modes, we can construct the monopole as

$$
E_{m}(r, \theta, \phi)=2 P_{0}(r, \theta, \phi),
$$

and the orthogonal dipoles as

$$
\begin{aligned}
E_{d}^{0}(r, \theta, \phi) & =2\left[P_{1}(r, \theta, \phi)+P_{2}(r, \theta, \phi)\right], \\
E_{d}^{\pi / 2}(r, \theta, \phi) & =2 j\left[P_{1}(r, \theta, \phi)-P_{2}(r, \theta, \phi)\right] .
\end{aligned}
$$

In matrix notation

$$
\left[\begin{array}{c}
E_{m} \\
E_{d}^{0} \\
E_{d}^{\pi / 2}
\end{array}\right]=\frac{2}{3}\left[\begin{array}{ccc}
1 & 1 & 1 \\
2 & -1 & -1 \\
0 & \sqrt{3} & -\sqrt{3}
\end{array}\right]\left[\begin{array}{c}
E_{c}^{0} \\
E_{c}^{1} \\
E_{c}^{2}
\end{array}\right] .
$$

For frequencies with wavelengths larger than the size of the array (for wavelengths smaller than the size of the array, spatial aliasing effects will occur), that is, $r \ll c / f$, the phase-component $\psi_{n}$, given by (5) can be neglected and the responses of the eigenbeams for these frequencies are equal to

$$
\begin{gathered}
E_{m}=, 1 \\
E_{d}^{0}(\theta, \phi)=\cos \phi \sin \theta, \\
E_{d}^{\pi / 2}(\theta, \phi)=\cos \left(\phi-\frac{\pi}{2}\right) \sin \theta .
\end{gathered}
$$

The directivity patterns of these eigenbeams are shown in Figure 2.

The zeroth-order eigenbeam $E_{m}$ represents the monopole response, while the first-order eigenbeams $E_{d}^{0}(\theta, \phi)$ and $E_{d}^{\pi / 2}(\theta, \phi)$ represent the orthogonal dipole responses.

The dipole can be steered to any angle $\varphi_{s}$ by means of a weighted combination of the orthogonal dipole pair:

$$
E_{d}^{\varphi_{s}}(\theta, \phi)=\cos \varphi_{s} E_{d}^{0}(\theta, \phi)+\sin \varphi_{s} E_{d}^{\pi / 2}(\theta, \phi),
$$

with $0 \leq \varphi_{s} \leq 2 \pi$ being the steering angle.

Finally, the steered and scaled superdirectional microphone response can be constructed via

$$
\begin{aligned}
E(\theta, \phi) & =S\left[\alpha E_{m}+(1-\alpha) E_{d}^{\varphi_{s}}(\theta, \phi)\right] \\
& =S\left[\alpha+(1-\alpha) \cos \left(\phi-\varphi_{s}\right) \sin \theta\right],
\end{aligned}
$$

with $\alpha \leq 1$ being the parameter for controlling the directional pattern of the first-order response and $S$ being an arbitrary scaling factor. Both parameters $\alpha$ and $S$ may also have negative values.

Alternatively, we can write the construction of the response in matrix-vector notation:

$$
E(\theta, \phi)=S \mathbf{F}_{\alpha}^{T} \mathbf{R}_{\varphi_{s}} \mathbf{X}
$$

with the pattern-synthesis vector:

$$
\mathbf{F}_{\alpha}=\left[\begin{array}{c}
\alpha \\
(1-\alpha) \\
0
\end{array}\right]
$$


the rotation-matrix $\mathbf{R}_{\varphi_{s}}$ :

$$
\mathbf{R}_{\varphi_{s}}=\left[\begin{array}{ccc}
1 & 0 & 0 \\
0 & \cos \varphi_{s} & \sin \varphi_{s} \\
0 & -\sin \varphi_{s} & \cos \varphi_{s}
\end{array}\right]
$$

and the input-vector:

$$
\mathbf{X}=\left[\begin{array}{c}
E_{m} \\
E_{d}^{0}(\theta, \phi) \\
E_{d}^{\pi / 2}(\theta, \phi)
\end{array}\right]=\left[\begin{array}{c}
1 \\
\cos \phi \sin \theta \\
\sin \phi \sin \theta
\end{array}\right] .
$$

In the remainder of this paper, we will assume that we have unity response of the superdirectional microphone for a desired source coming from an arbitrary azimuthal angle $\phi=\varphi_{s}$ and for an elevation angle $\theta=\pi / 2$ and we want to suppress two interferers by steering two nulls towards two azimuthal angles $\phi=\varphi_{n_{1}}$ and $\phi=\varphi_{n_{2}}$, also for an elevation angle $\theta=\pi / 2$. Hence, we assume $\theta=\pi / 2$ in the remainder of this paper.

\section{Optimal Null-Steering for Two Directional Interferers via Direct Pattern Synthesis}

3.1. Pattern Synthesis. The first-order response of (12), with the main-lobe of the response steered to $\varphi_{s}$, has two nulls for $\alpha \leq 1 / 2$, given by (see [13])

$$
\varphi_{n_{1}}, \varphi_{n_{2}}=\varphi_{s} \pm \arccos \left(\frac{-\alpha}{1-\alpha}\right) .
$$

If we want to steer two nulls to arbitrary angles $\varphi_{n_{1}}$ and $\varphi_{n_{2}}$, not lying symmetrical with respect to $\varphi_{s}$, it can be seen that we cannot steer the main-lobe of the first-order response to $\varphi_{s}$. Therefore, we steer the main-lobe to $\tilde{\varphi}_{s}$ and use a scalefactor $\tilde{S}$ under the constraint that a unity response is obtained at angle $\varphi_{s}$. In matrix notation,

$$
E(\theta, \phi)=\widetilde{S}_{\widetilde{\alpha}}^{T} \mathbf{R}_{\tilde{\varphi}_{s}} \mathbf{X},
$$

with the rotation-matrix and the pattern-synthesis matrix being as in (15) and (14), respectively, with $\tilde{\alpha}, \tilde{\varphi}_{s}$ instead of $\alpha, \varphi_{s}$.

From (12), we see that a unity desired response at angle $\varphi_{s}$ is obtained when we choose the scale-factor $\tilde{S}$ as

$$
\widetilde{S}=\frac{1}{\tilde{\alpha}+(1-\tilde{\alpha}) \cos \left(\varphi_{s}-\tilde{\varphi}_{s}\right)}
$$

with $\tilde{\alpha}$ being the parameter for controlling the directional pattern of the first-order response (similar to the parameter $\alpha), \varphi_{s}$ the angle for the desired sound, and $\tilde{\varphi}_{s}$ the angle for the steering (which, in general, is different from $\varphi_{s}$ ).

Next, we want to place the nulls at $\varphi_{n_{1}}$ and $\varphi_{n_{2}}$. Hence, we solve the following system of two equations:

$$
\begin{aligned}
& \tilde{S}\left[\tilde{\alpha}+(1-\tilde{\alpha}) \cos \left(\varphi_{n_{1}}-\tilde{\varphi}_{s}\right)\right]=0, \\
& \tilde{S}\left[\tilde{\alpha}+(1-\tilde{\alpha}) \cos \left(\varphi_{n_{2}}-\tilde{\varphi}_{s}\right)\right]=0 .
\end{aligned}
$$

Solving the two unknowns $\tilde{\alpha}$ and $\tilde{\varphi}_{s}$ gives

$$
\begin{gathered}
\tilde{\varphi}_{s}=2 \arctan X, \\
\tilde{\alpha}=\frac{\sin \left(\Delta \varphi_{n}\right) X}{\cos \varphi_{n_{1}}-\cos \varphi_{n_{2}}+X\left[\sin \varphi_{n_{1}}-\sin \varphi_{n_{2}}+\sin \left(\Delta \varphi_{n}\right)\right]},
\end{gathered}
$$

with

$$
\begin{gathered}
X=\frac{\sin \varphi_{n_{1}}-\sin \varphi_{n_{2}} \pm \sqrt{2-2 \cos \left(\Delta \varphi_{n}\right)}}{\cos \varphi_{n_{1}}-\cos \varphi_{n_{2}}}, \\
\Delta \varphi_{n}=\varphi_{n_{1}}-\varphi_{n_{2}} .
\end{gathered}
$$

It is noted that (23) can have two solutions, leading to different solutions for $\tilde{\varphi}_{s}, \tilde{\alpha}$, and $\tilde{S}$. However, the resulting beampatterns are identical.

As can be seen we get a vanishing denominator in (22) for $\varphi_{n_{1}}=\varphi_{s}$ and/or $\varphi_{n_{2}}=\varphi_{s}$. Similarly, this is the case when $\Delta \varphi_{n}=\varphi_{n_{1}}-\varphi_{n_{2}}$ goes to zero. For this latter case, we can compute the limit of $\tilde{\varphi}_{s}$ and $\tilde{\alpha}$ :

$$
\lim _{\Delta \varphi_{n} \rightarrow 0} \tilde{\varphi}_{s}=2 \arctan \left[\frac{\sin \varphi_{n_{i}}}{\cos \varphi_{n_{i}}-1}\right]=\varphi_{n_{i}}+\pi,
$$

with $i=1,2$ and

$$
\lim _{\Delta \varphi_{n} \rightarrow 0} \tilde{\alpha}=\frac{1}{2}
$$

where $\Delta \varphi_{n}=\varphi_{n_{1}}-\varphi_{n_{2}}$.

For the case $\Delta \varphi_{n}=0$, we actually steer a single null towards the two directional interferers $\varphi_{n_{1}}$ and $\varphi_{n_{2}}$. Equations (25) and (26) describe the limit-case solution for which there are an infinite number of solutions that satisfy the system of equations, given by (21).

3.2. Analysis of Directivity Index. Although the optimization in this paper is focused on the suppression of two directional interferers, it is also important to analyze the noise-reduction performance for isotropic noise circumstances. We will only analyze the spherical isotropic noise case, for which we compute the spherical directivity factor $Q_{S}$ given by $[4,5]$

$$
Q_{S}=\frac{4 \pi E^{2}\left(\pi / 2, \varphi_{s}\right)}{\int_{\phi=0}^{2 \pi} \int_{\theta=0}^{\pi} E^{2}(\theta, \phi) \sin \theta d \theta d \phi} .
$$

If we combine (27) with (18), we get

$$
Q_{S}\left(\varphi_{1}, \varphi_{2}\right)=\frac{6\left(1-\cos \varphi_{1}\right)\left(1-\cos \varphi_{2}\right)}{5+3 \cos \left(\varphi_{1}-\varphi_{2}\right)},
$$

with

$$
\begin{aligned}
& \varphi_{1}=\varphi_{n_{1}}-\varphi_{s}, \\
& \varphi_{2}=\varphi_{n_{2}}-\varphi_{s} .
\end{aligned}
$$

In Figure 3, the contour-plot of the directivity factor $Q_{S}$ is shown with $\varphi_{1}$ and $\varphi_{2}$ on the $x$ - and $y$-axes, respectively. 


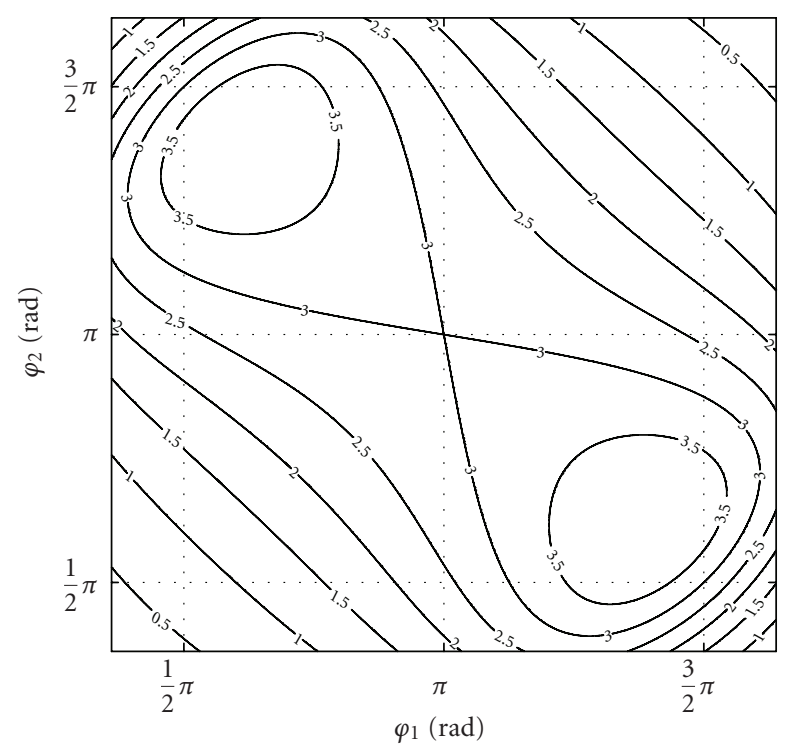

FIgURE 3: Contour-plot of the directivity factor $Q_{S}\left(\varphi_{1}, \varphi_{2}\right)$.

As can be seen in (28), the directivity factor goes to zero if one of the angles $\varphi_{n_{1}}$ or $\varphi_{n_{2}}$ gets close to $\varphi_{s}$. Clearly, a directivity factor which is smaller than unity is not very useful in practice. Hence, the pattern synthesis technique is only useful when the angles $\varphi_{n_{1}}$ and $\varphi_{n_{2}}$ are located in one half-plane and the desired source is located around the center of the opposite half-plane.

It can be found in the appendix that for

$$
\begin{gathered}
\varphi_{1}=\arccos \left(-\frac{1}{3}\right), \\
\varphi_{2}=2 \pi-\arccos \left(-\frac{1}{3}\right),
\end{gathered}
$$

a maximum directivity factor $Q_{S}=4$ is obtained. This corresponds with $6 \mathrm{~dB}$ directivity index, defined as $10 \log _{10} Q_{S}$, where the directivity pattern resembles a hypercardioid. Furthermore for $\left(\varphi_{1}, \varphi_{2}\right)=(\pi, \pi)$ rad. a directivity factor $Q_{S}=3$ is obtained, corresponding with $4.8 \mathrm{~dB}$ directivity index, where the directivity pattern yields a cardioid. As can be seen from Figure 3, we can define a usable region, where the directivity-factor is $Q_{S}>3 / 4$ for $\pi / 2 \leq \varphi_{1}, \varphi_{2} \leq 3 \pi / 2$.

\section{Optimal Null-Steering for Two Directional Interferers via GSC}

4.1. Generalized Sidelobe Canceller (GSC) Structure. To develop an adaptive algorithm for steering two nulls towards the two directional interferers based on the pattern-synthesis technique in Section 3, it would be required to use a constrained optimization technique where we want to maintain a unity response towards the angle $\varphi_{s}$. For adaptive algorithms, it is generally easier to adapt in an unconstrained manner. Therefore, we first present an alternative scheme for the null-steering, similar to the direct pattern-synthesis technique as discussed in Section 3, but based on the wellknown Generalized Sidelobe Canceller (GSC) [12]. In the

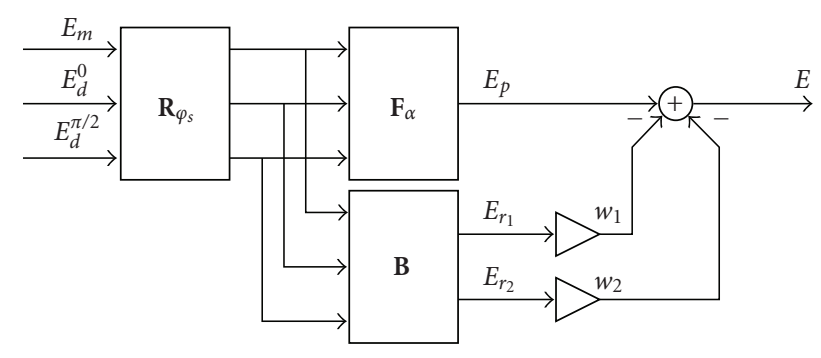

Figure 4: Generalized Sidelobe Canceller scheme.

GSC scheme, first a prefiltering with a fixed value of $\varphi_{s}$ and $\alpha$ is performed, to construct a primary signal with a unity response to angle $\varphi_{s}$ and two noise references. As the two noise references do not include the source coming from angle $\varphi_{s}$, two noise-canceller weights $w_{1}$ and $w_{2}$ can be optimized in an unconstrained manner. The GSC scheme is shown in Figure 4.

We start by constructing the primary-response as

$$
E_{p}(\theta, \phi)=\mathbf{F}_{\alpha}^{T} \mathbf{R}_{\varphi_{s}} \mathbf{X}
$$

with $\mathbf{F}_{\alpha}^{T}, \mathbf{R}_{\varphi_{s}}$, and $\mathbf{X}$ being as defined in the introduction and using a scale-factor $S=1$.

Furthermore, we can create two noise-references via

$$
\left[\begin{array}{c}
E_{r_{1}}(\theta, \phi) \\
E_{r_{2}}(\theta, \phi)
\end{array}\right]=\mathbf{B}^{T} \mathbf{R}_{\varphi_{s}} \mathbf{X}
$$

with a blocking-matrix $\mathbf{B}$ [14] given by

$$
\mathbf{B}=\left[\begin{array}{cc}
\frac{1}{2} & 0 \\
-\frac{1}{2} & 0 \\
0 & 1
\end{array}\right] \text {. }
$$

It is noted that the noise-references $E_{r_{1}}$ and $E_{r_{2}}$ are, respectively, a cardioid and a dipole response, with a null steered towards the angle of the desired source at azimuth $\phi=\varphi_{s}$ and elevation $\theta=\pi / 2$.

The primary- and the noise-responses can be used in the generalized sidelobe canceller structure, to obtain an output as

$$
E(\theta, \phi)=E_{p}(\theta, \phi)-w_{1} E_{r_{1}}(\theta, \phi)-w_{2} E_{r_{2}}(\theta, \phi) .
$$

It is important to note that for any value of $\varphi_{s}, \alpha, w_{1}$, and $w_{2}$, a unity-response at the output of the GSC is maintained for angle $\phi=\varphi_{s}$ and $\theta=\pi / 2$.

In the next sections we give some details in computing $w_{1}$ and $w_{2}$ for the suppression of two directional interferers, as discussed in the previous section.

4.2. Optimal GSC Null-Steering for Two Directional Interferers. Using the GSC structure of Figure 4 having a unity response at angle $\phi=\varphi_{s}$, we can compute the weights $w_{1}$ 
and $w_{2}$ to steer two nulls towards azimuthal angles $\varphi_{n_{1}}$ and $\varphi_{n_{2}}$, by solving

$$
E_{p}\left(\frac{\pi}{2}, \varphi_{i}\right)-w_{1} E_{r_{1}}\left(\frac{\pi}{2}, \varphi_{i}\right)-w_{2} E_{r_{2}}\left(\frac{\pi}{2}, \varphi_{i}\right)=0
$$

for $i=1,2$.

This results in the following relations:

$$
\begin{gathered}
w_{1}=2 \alpha+\frac{2 \sin \left(\varphi_{1}-\varphi_{2}\right)}{\sin \varphi_{1}-\sin \left(\varphi_{1}-\varphi_{2}\right)-\sin \varphi_{2}}, \\
w_{2}=\frac{\cos \varphi_{1}-\cos \varphi_{2}}{\sin \varphi_{1}-\sin \left(\varphi_{1}-\varphi_{2}\right)-\sin \varphi_{2}},
\end{gathered}
$$

where $\varphi_{1}$ and $\varphi_{2}$ are defined as given by (29) and (30), respectively.

To eliminate the dependency of $\alpha$ in (37), we will use

$$
\widetilde{w}_{1}=w_{1}-2 \alpha \text {. }
$$

The denominators in (37) and (38) vanish when $\varphi_{n_{1}}=\varphi_{s}$ and/or $\varphi_{n_{2}}=\varphi_{s}$. Also when $\Delta \varphi_{n}=\varphi_{n_{1}}-\varphi_{n_{2}}$ goes to zero, the denominator vanishes. In this case, we can compute the limit of $\tilde{w}_{1}$ and $w_{2}$ :

$$
\begin{gathered}
\lim _{\Delta \varphi_{n} \rightarrow 0} \tilde{w}_{1}=-2, \\
\lim _{\Delta \varphi_{n} \rightarrow 0} \mathcal{w}_{2}=\sin \varphi_{i}
\end{gathered}
$$

with $i=1,2$.

For the case $\Delta \varphi_{n}=0$, we actually steer a single null towards the two directional interferers $\varphi_{n_{1}}$ and $\varphi_{n_{2}}$. Equations (40) and (41) describe the limit-case solution for which there are an infinite number of solutions $\left(\widetilde{w}_{1}, w_{2}\right)$ that satisfy $(36)$.

From the values of $\tilde{w}_{1}$ and $w_{2}$, we can derive the two angles of the directional interferers $\vartheta_{1}$ and $\vartheta_{2}$, where $\left(\vartheta_{1}, \vartheta_{2}\right)=\left(\varphi_{1}, \varphi_{2}\right)$ or $\left(\vartheta_{1}, \vartheta_{2}\right)=\left(\varphi_{2}, \varphi_{1}\right)$. The two angles are obtained via a computation involving the arctan-function with additional sign checking to resolve all four quadrants in the azimuthal plane and can be computed as

$$
\vartheta_{1}, \vartheta_{2}= \begin{cases}\arctan \left(\frac{N}{D}\right) & \text { for : } D \geq 0, \\ \arctan \left(\frac{N}{D}\right)+\pi & \text { for : } D<0, N \geq 0, \\ \arctan \left(\frac{N}{D}\right)-\pi & \text { for : } D<0, N<0,\end{cases}
$$

with

$$
\begin{gathered}
N=\frac{-2\left(\tilde{w}_{1} w_{2} \mp X_{1}\right)}{X_{2}}, \\
D=\frac{\widetilde{w}_{1}^{3}+4 \tilde{w}_{1}^{2}+4 \tilde{w}_{1} \pm 4 w_{2} X_{1}}{X_{2}\left(\tilde{w}_{1}+2\right)},
\end{gathered}
$$

with

$$
\begin{gathered}
X_{1}=\sqrt{\left(\tilde{w}_{1}+2\right)^{2}\left(1+\tilde{w}_{1}+w_{2}^{2}\right)}, \\
X_{2}=4+4 \widetilde{w}_{1}+\widetilde{w}_{1}^{2}+4 w_{2}^{2} .
\end{gathered}
$$

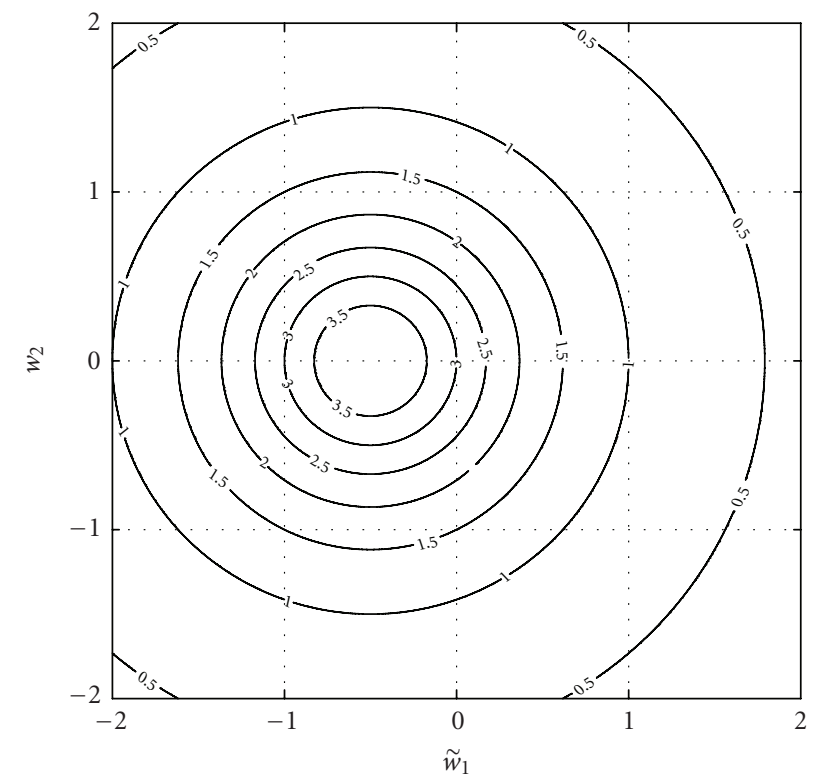

FIGURE 5: Contour-plot of the directivity factor $Q_{S}\left(\widetilde{w}_{1}, w_{2}\right)$.

Note that with this computation, it is not necessarily true that $\vartheta_{1}=\varphi_{1}$ and $\vartheta_{2}=\varphi_{2}$, that is, we can have a permutation ambiguity. Furthermore, we compute the resolved angles of the directional interferers as

$$
\vartheta_{n_{i}}=\vartheta_{i}-\varphi_{s}
$$

where $\left(\vartheta_{n_{1}}, \vartheta_{n_{2}}\right)=\left(\varphi_{n_{1}}, \varphi_{n_{2}}\right)$ or $\left(\vartheta_{n_{1}}, \vartheta_{n_{2}}\right)=\left(\varphi_{n_{2}}, \varphi_{n_{1}}\right)$.

4.3. Analysis of Directivity Index. Just as for the direct pattern synthesis in the previous section, we can analyze the directivity factor for spherical isotropic noise. We can insert the values of $w_{1}$ and $w_{2}$ into (27) and (35) and get

$$
Q_{S}\left(\widetilde{w}_{1}, w_{2}\right)=\frac{3}{\widetilde{w}_{1}+\widetilde{w}_{1}^{2}+w_{2}^{2}+1}
$$

In Figure 5, we show the contour-plot of the directivity factor with $\widetilde{w}_{1}$ and $w_{2}$ on the $x$-and $y$-axes, respectively.

From Figure 5 and (46), it can be seen that the contours are concentric circles with the center at coordinate $\left(\tilde{w}_{1}, w_{2}\right)=$ $(-1 / 2,0)$ where the maximum directivity factor of 4 is obtained.

\section{Adaptive Algorithm}

5.1. Cost-Function for Directional Interferers. Next, we develop an adaptation scheme to adapt two weights in the GSC structure as discussed in the previous Section 4 . We aim at obtaining the solution, where a unity response is obtained at angle $\varphi_{s}$ and two nulls are placed at angles $\varphi_{n_{1}}$ and $\varphi_{n_{2}}$.

We start with

$$
y[k]=p[k]-\left(\widehat{w}_{1}[k]+2 \alpha\right) r_{1}[k]-\widehat{w}_{2}[k] r_{2}[k],
$$

with $k$ being the discrete-time index, $y[k]$ the output signal, $\widehat{w}_{1}[k]$ and $\widehat{w}_{2}[k]$ the adaptive weights, $r_{1}[k]$ and $r_{2}[k]$ the 
noise reference signals, and $p[k]$ the primary signal. The inclusion of the term $2 \alpha$ in (47) is a consequence of the fact that $\hat{w}_{1}[k]$ is an estimate of $\tilde{w}_{1}$ (see (39) in which $2 \alpha$ is not included).

In the ideal case that we want to obtain a unity response for a source-signal $s[k]$ originating from angle $\varphi_{s}$ and have an undesired source-signal $n_{1}[k]$ originating from angle $\varphi_{n_{1}}$ together with an undesired source-signal $n_{2}[k]$ originating from angle $\varphi_{n_{2}}$, we have

$$
\begin{gathered}
p[k]=s[k]+\sum_{i=1,2}\left[\alpha+(1-\alpha) \cos \varphi_{i}\right] n_{i}[k] \\
r_{1}[k]=\sum_{i=1,2}\left(\frac{1}{2}-\frac{1}{2} \cos \varphi_{i}\right) n_{i}[k] \\
r_{2}[k]=\sum_{i=1,2} \sin \varphi_{i} n_{i}[k] .
\end{gathered}
$$

The cost-function $J\left(\widehat{w}_{1}, \widehat{w}_{2}\right)$ is defined as a function of $\widehat{w}_{1}$ and $\widehat{w}_{2}$ and is given by

$$
J\left(\widehat{w}_{1}, \widehat{w}_{2}\right)=\mathscr{E}\left\{y^{2}[k]\right\},
$$

with $\&\{\cdot\}$ being the expectation operator.

Using that $\mathscr{E}\left\{n_{1}[k] n_{2}[k]\right\}=0$ and $\mathscr{E}\left\{n_{i}[k] s[k]\right\}=0$ for $i=1,2$, we can write

$$
\begin{aligned}
J\left(\widehat{w}_{1}, \widehat{w}_{2}\right)= & \mathcal{E}\left\{\left[p[k]-\left(\hat{w}_{1}[k]+2 \alpha\right) r_{1}[k]-\widehat{w}_{2}[k] r_{2}[k]\right]^{2}\right\} \\
= & \sigma_{s}^{2}[k]+\sum_{i=1,2} \sigma_{n_{i}}^{2}[k] \\
\times & {\left[\frac{1}{4} \widehat{w}_{1}[k]^{2}+\widehat{w}_{2}[k]^{2}\right.} \\
& +\cos ^{2} \varphi_{i}\left(\frac{1}{4} \widehat{w}_{1}[k]^{2}+\widehat{w}_{1}[k]-\widehat{w}_{2}[k]^{2}+1\right) \\
& +\cos \varphi_{i}\left(-\frac{1}{2} \widehat{w}_{1}[k]^{2}-\widehat{w}_{1}[k]\right)+\sin \varphi_{i} \widehat{w}_{1}[k] \widehat{w}_{2}[k] \\
& \left.+\cos \varphi_{i} \sin \varphi_{i}\left(-2 \widehat{w}_{2}[k]-\widehat{w}_{1}[k] \widehat{w}_{2}[k]\right)\right] \\
= & \sigma_{s}^{2}[k]+\sum_{i=1,2}\left[\widehat{w}_{1}[k]-\left(2+\widehat{w}_{1}[k]\right) \cos \varphi_{i}\right. \\
& \left.+2 \widehat{w}_{2}[k] \sin \varphi_{i}\right]^{2} \frac{\sigma_{n_{i}}^{2}[k]}{4},
\end{aligned}
$$

with

$$
\begin{aligned}
\sigma_{s}^{2}[k] & =\mathscr{E}\left\{s^{2}[k]\right\}, \\
\sigma_{n_{i}}^{2}[k] & =\mathscr{E}\left\{n_{i}^{2}[k]\right\} .
\end{aligned}
$$

We can see that the cost-function is a quadratic-function [15] that can be written in matrix-notation (for convenience, we leave out the index $k$ ):

$$
\begin{aligned}
J\left(\widehat{w}_{1}, \hat{w}_{2}\right) & =\sigma_{s}^{2}+\left\|\mathbf{A}_{p} \underline{\hat{w}}-\underline{v}_{p}\right\|^{2} \\
& =\sigma_{s}^{2}+\underline{w}^{T} \mathbf{A}_{p}^{T} \mathbf{A}_{p} \underline{w}-2 \underline{w}^{T} \mathbf{A}_{p}^{T} \underline{v}_{p}+\underline{v}_{p}^{T} \underline{v}_{p}
\end{aligned}
$$

with

$$
\begin{gathered}
\mathbf{A}_{p}=\left[\begin{array}{cc}
\frac{\sigma_{n_{1}}}{2}\left(1-\cos \varphi_{1}\right) & \sigma_{n_{1}} \sin \varphi_{1} \\
\frac{\sigma_{n_{2}}}{2}\left(1-\cos \varphi_{2}\right) & \sigma_{n_{2}} \sin \varphi_{2}
\end{array}\right], \\
\underline{\hat{w}}=\left[\begin{array}{l}
\widehat{w}_{1} \\
\widehat{w}_{2}
\end{array}\right], \\
\underline{v}_{p}=\left[\begin{array}{l}
\sigma_{n_{1}} \cos \varphi_{1} \\
\sigma_{n_{2}} \cos \varphi_{2}
\end{array}\right] .
\end{gathered}
$$

The singularity of $\mathbf{A}_{p}^{T} \mathbf{A}_{p}$ can be analyzed by computing the determinant of $\mathbf{A}_{p}$ and setting this determinant to zero:

$$
\frac{\sigma_{n_{1}} \sigma_{n_{2}}}{2}\left[\sin \varphi_{2}\left(1-\cos \varphi_{1}\right)-\sin \varphi_{1}\left(1-\cos \varphi_{2}\right)\right]=0 .
$$

Equation (54) is satisfied when $\sigma_{n_{1}}$ and/or $\sigma_{n_{2}}$ are equal to zero, $\varphi_{1}$ and/or $\varphi_{2}$ are equal to zero, or when

$$
\frac{\sin \varphi_{1}}{1-\cos \varphi_{1}}=\frac{\sin \varphi_{2}}{1-\cos \varphi_{2}} \equiv \cot \left(\frac{\varphi_{1}}{2}\right)=\cot \left(\frac{\varphi_{2}}{2}\right) .
$$

Equation (55) is satisfied only when $\varphi_{1}=\varphi_{2}$. This agrees with the result that was obtained in Section 3.1, where $\Delta \varphi=$ 0 .

In all other cases (so when $\varphi_{1} \neq \varphi_{2}, \sigma_{n_{1}}>0$ and $\sigma_{n_{2}}>0$ ), the matrix $\mathbf{A}_{p}$ is nonsingular and the matrix $\mathbf{A}_{p}^{T} \mathbf{A}_{p}$ is positive definite. Hence, the cost-function is a convex function with a global minimum that can be found by solving the leastsquares problem:

$$
\begin{aligned}
\widehat{\underline{w}}_{\mathrm{opt}} & =\left(\mathbf{A}_{p}^{T} \mathbf{A}_{p}\right)^{-1} \mathbf{A}_{p}^{T} \underline{v}_{p} \\
& =\mathbf{A}_{p}^{-1} \underline{v}_{p} \\
& =\frac{1}{A}\left[\begin{array}{c}
2 \sin \left(\varphi_{1}-\varphi_{2}\right) \\
\cos \varphi_{1}-\cos \varphi_{2}
\end{array}\right],
\end{aligned}
$$

with

$$
A=\sin \varphi_{1}-\sin \left(\varphi_{1}-\varphi_{2}\right)-\sin \varphi_{2},
$$

similar to the solutions as given in (37) and (38).

As an example, we show the contour-plot of the costfunction $10 \log _{10} J\left(\widehat{w}_{1}, \widehat{w}_{2}\right)$ in Figure 6 , for the case where $\varphi_{s}=\pi / 2, \varphi_{n_{1}}=0, \varphi_{n_{2}}=\pi \operatorname{rad} ., \sigma_{n_{i}}^{2}=1$ for $i=1,2$, and $\sigma_{s}^{2}=0$.

As can be seen, the global minimum is obtained for $\widehat{w}_{1}=$ 0 and $\widehat{w}_{2}=0$, resulting in a dipole beampattern. When we change $\sigma_{n_{1}}^{2} \neq \sigma_{n_{2}}^{2}$, the shape of the cost-function will be more and more stretched, but the global optimum will be obtained for the same values of $\widehat{w}_{1}$ and $\widehat{w}_{2}$. In the extreme case when $\sigma_{n_{2}}^{2}=0$ and $\sigma_{n_{1}}^{2}>0$, we obtain the cost-function as shown in Figure 7. (It is interesting to note that this cost-function is exactly the same as for the case where $\varphi_{s}=\pi / 2, \varphi_{n_{1}}=\varphi_{n_{2}}=0$ radians with $\sigma_{n_{i}}^{2}=1$ for $i=1,2$ and $\sigma_{s}^{2}=0$.) Although still $\hat{w}_{1}=0$ and $\hat{w}_{2}=0$ is an optimal solution, it can be 


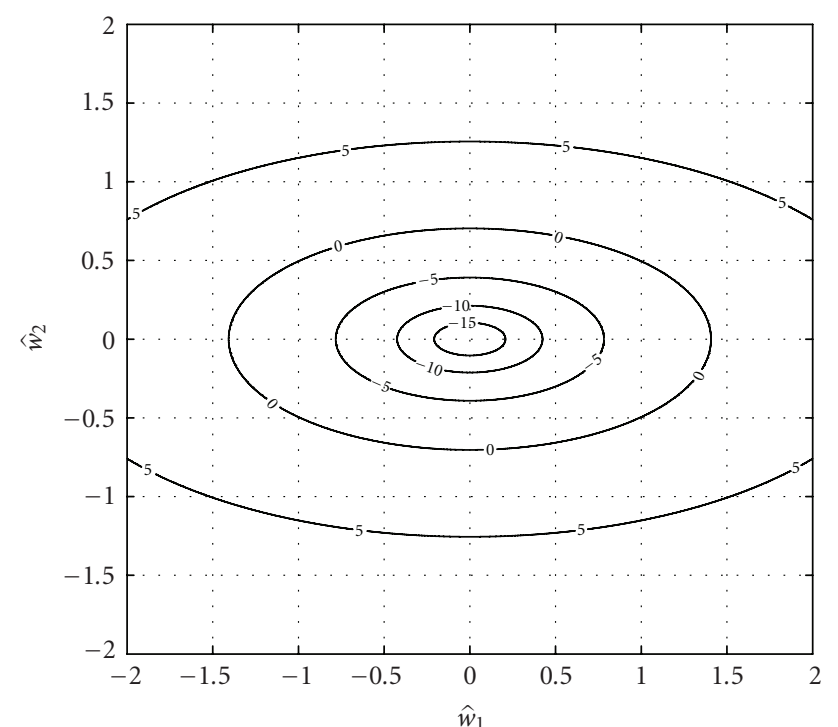

Figure 6: Contour-plot of the cost-function $10 \log _{10} J\left(\widehat{w}_{1}, \widehat{w}_{2}\right)$ for the case where $\varphi_{s}=\pi / 2, \varphi_{n_{1}}=0$, and $\varphi_{n_{2}}=\pi$ radians.

seen that there is no strict global minimum. For example, also $\hat{w}_{1}=-2$ and $\hat{w}_{2}=1$ is an optimal solution (yielding a cardioid beampattern).

For the situation where there is only a single interferer or the situation where there are two interferers coming from (nearly) the same angle, the resulting beampattern will have a null to this angle, while the other (second) null will be placed randomly (i.e., the second null is not uniquely defined and the adaptation of this second null is poor). However in situations where we have additive diffuse-noise present, we obtain an extra degree of freedom, for example, optimization of the directivity index. This is however outside the scope of this paper.

5.2. Cost-Function for Isotropic Noise. It is also useful to analyze the cost-function in the presence of isotropic (i.e., diffuse) noise. We know from [16] that spherical and cylindrical isotropic noise can be modelled by adding uncorrelated additive white-noise signals $d_{1}, d_{2}$, and $d_{3}$ to the three eigenbeams $E_{m}, E_{d}^{0}$, and $E_{d}^{\pi / 2}$ with variances $\sigma_{d}^{2}, \sigma_{d}^{2} \gamma$, and $\sigma_{d}^{2} \gamma$, respectively, or alternatively with a covariance matrix $\mathbf{K}_{d}$ given by

$$
\mathbf{K}_{d}=\sigma_{d}^{2}\left[\begin{array}{lll}
1 & 0 & 0 \\
0 & \gamma & 0 \\
0 & 0 & \gamma
\end{array}\right] .
$$

(for diffuse noise situations, the individual elements are correlated. However, due the construction of eigenbeams, the diffuse noise will be decorrelated. Hence, it is allowed to add uncorrelated additive white-noise signals to these eigenbeams to simulate diffuse-noise situations,) We choose $\gamma=1 / 3$ for spherically isotropic noise and $\gamma=1 / 2$ for cylindrically isotropic noise.

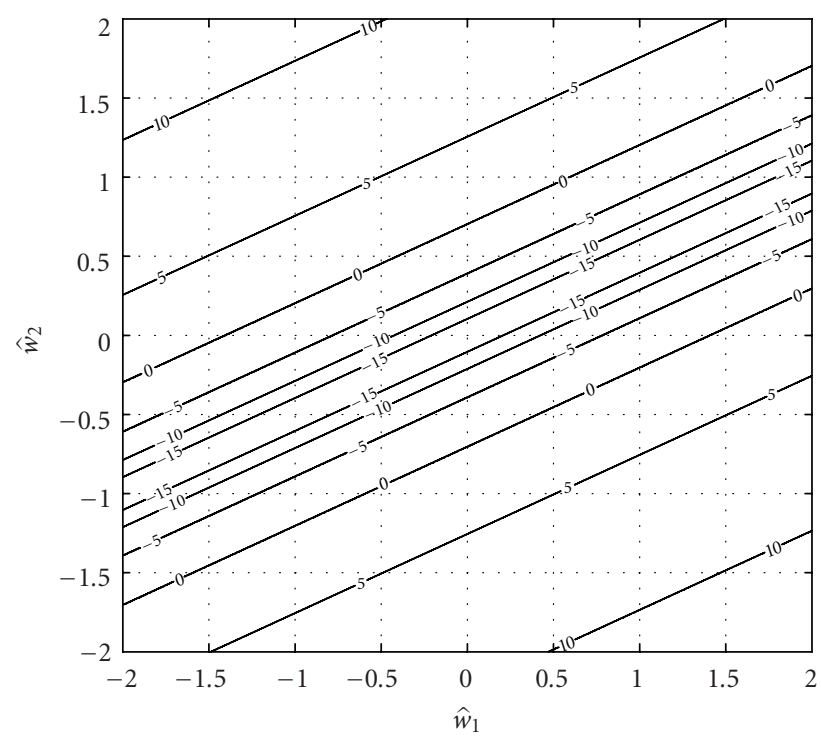

Figure 7: Contour-plot of the cost-function $10 \log _{10} J\left(\widehat{w}_{1}, \widehat{w}_{2}\right)$ for the case where $\varphi_{s}=\pi / 2$ and $\varphi_{n_{1}}=\varphi_{n_{2}}=0$ radians.

Assuming that there are no directional interferers, we obtain the following primary signal $p[k]$ and noisereferences $r_{1}[k]$ and $r_{2}[k]$ in the generalized sidelobe canceller scheme:

$$
\begin{gathered}
p[k]=s[k]+\alpha d_{1}[k]+(1-\alpha) d_{2}[k] \sqrt{\gamma}, \\
r_{1}[k]=\frac{1}{2} d_{1}[k]-\frac{1}{2} d_{2}[k] \sqrt{\gamma}, \\
r_{2}[k]=d_{3}[k] \sqrt{\gamma} .
\end{gathered}
$$

As $d_{i}[k]$ with $i=1,2,3$ and $s[k]$ are mutually uncorrelated, we can write the cost-function as

$$
J\left(\widehat{w}_{1}, \widehat{w}_{2}\right)=\sigma_{s}^{2}[k]+\sigma_{d}^{2}\left[\left(\frac{1}{2} \widehat{w}_{1}\right)^{2}+\gamma\left(1+\frac{1}{2} \widehat{w}_{1}\right)^{2}+\gamma \widehat{w}_{2}^{2}\right] .
$$

Just as for the cost-function with two directional interferers, we can write the cost-function for isotropic noise also as a quadratic function in matrix notation:

$$
J_{d}\left(\widehat{w}_{1}, \widehat{w}_{2}\right)=\sigma_{s}^{2}+\left[\left\|\mathbf{A}_{d} \underline{\hat{w}}-\underline{v}_{d}\right\|^{2}+\frac{\gamma}{1+\gamma}\right],
$$

with

$$
\begin{gathered}
\mathbf{A}_{d}=\left[\begin{array}{cc}
\frac{\sigma_{d}}{2} \sqrt{1+\gamma} & 0 \\
0 & \sigma_{d \sqrt{\gamma}}
\end{array}\right], \\
\underline{v}_{d}=\left[\begin{array}{c}
\frac{-\sigma_{d} \gamma}{\sqrt{1+\gamma}} \\
0
\end{array}\right] .
\end{gathered}
$$


It can be easily seen that $\mathbf{A}_{d}$ is positive definite and hence we have a convex cost-function with a global minimum. Via (56) we can easily compute this minimum of the costfunction, which is obtained by solving the least-squares problem:

$$
\begin{aligned}
\widehat{\underline{w}}_{\mathrm{opt}} & =\left(\mathbf{A}_{d}^{T} \mathbf{A}_{d}\right)^{-1} \mathbf{A}_{d}^{T} \underline{v}_{d} \\
& =\mathbf{A}_{p}^{-1} \underline{v}_{p} \\
& =\left[\begin{array}{c}
-\frac{2 \gamma}{1+\gamma} \\
0
\end{array}\right] .
\end{aligned}
$$

5.3. Cost-Function for Directional Interferers and Isotropic Noise. In case we have directional interferers as well as isotropic noise and assume that all these noise-components are mutually uncorrelated, we can construct the costfunction based on addition of the two cost-functions:

$$
\begin{aligned}
& J_{p, d}\left(\widehat{w}_{1}, \widehat{w}_{2}\right)=J_{p}\left(\widehat{w}_{1}, \widehat{w}_{2}\right)+J_{d}\left(\widehat{w}_{1}, \widehat{w}_{2}\right) \\
& =\sigma_{s}^{2}+\left\|\mathbf{A}_{p} \underline{\hat{w}}-\underline{v}_{p}\right\|^{2}+\left\|\mathbf{A}_{d} \underline{\hat{w}}-\underline{v}_{d}\right\|^{2}+\frac{\sigma_{d}^{2} \gamma}{1+\gamma} \\
& =\sigma_{s}^{2}+\left\|\mathbf{A}_{p, d} \underline{\hat{w}}-\underline{v}_{p, d}\right\|^{2}+\frac{\sigma_{d}^{2} \gamma}{1+\gamma},
\end{aligned}
$$

with:

$$
\begin{aligned}
& \mathbf{A}_{p, d}=\left[\begin{array}{l}
\mathbf{A}_{p} \\
\mathbf{A}_{d}
\end{array}\right], \\
& \underline{v}_{p, d}=\left[\begin{array}{l}
\underline{v}_{p} \\
\underline{v}_{d}
\end{array}\right] .
\end{aligned}
$$

Since $J_{p}\left(\widehat{w}_{1}, \widehat{w}_{2}\right)$ and $J_{d}\left(\widehat{w}_{1}, \widehat{w}_{2}\right)$ were found to be convex, the sum $J_{p, d}\left(\hat{w}_{1}, \hat{w}_{2}\right)$ is also convex. The optimal weights $\underline{\hat{w}}_{\text {opt }}$ can be obtained by computing

$$
\widehat{\widehat{w}}_{\text {opt }}=\left(\mathbf{A}_{p, d}^{T} \mathbf{A}_{p, d}\right)^{-1} \mathbf{A}_{p, d}^{T} \underline{v}_{p, d},
$$

which can be solved numerically via standard SVD techniques [15].

5.4. Gradient Search Algorithm. As we know that the costfunction is a convex function with a global minimum, we can find this optimal solution by means of a steepest descent update equation for $\hat{w}_{i}$ with $i=1,2$ by stepping in the direction opposite to the surface $J\left(\widehat{w}_{1}, \widehat{w}_{2}\right)$ with respect to $\widehat{w}_{i}$, similar to [5]

$$
\widehat{w}_{i}[k+1]=\widehat{w}_{i}[k]-\mu \nabla_{\hat{w}_{i}} J\left(\widehat{w}_{1}, \widehat{w}_{2}\right),
$$

with a gradient given by

$$
\nabla_{\widehat{w}_{i}} J\left(\widehat{w}_{1}, \widehat{w}_{2}\right)=\frac{\partial J\left(\widehat{w}_{1}[k], \hat{w}_{2}[k]\right)}{\partial \widehat{w}_{i}[k]}=\frac{\partial \mathcal{E}\left\{y^{2}[k]\right\}}{\partial \widehat{w}_{i}[k]},
$$

and where $\mu$ is the update step-size. As in practice, the ensemble average $\mathcal{E}\left\{y^{2}[k]\right\}$ is not available, we have to use an instantaneous estimate of the gradient $\hat{\nabla}_{\hat{w}_{i}} J\left(\hat{w}_{1}, \hat{w}_{2}\right)$, which is computed as

$$
\begin{aligned}
\hat{\nabla}_{\hat{w}_{i}} J\left(\widehat{w}_{1}, \widehat{w}_{2}\right) & =\frac{d y^{2}[k]}{d \widehat{w}_{i}} \\
& =-2\left\{p[k]-\left(\widehat{w}_{1}+2 \alpha\right) r_{1}[k]-\widehat{w}_{2} r_{2}[k]\right\} r_{i}[k] \\
& =-2 y[k] r_{i}[k] .
\end{aligned}
$$

Hence, we can write the update equation as

$$
\widehat{w}_{i}[k+1]=\widehat{w}_{i}[k]+2 \mu y[k] r_{i}[k] .
$$

Just as proposed in [5], we can apply a powernormalization such that the convergence speed is independent of the power:

$$
\widehat{w}_{i}[k+1]=\widehat{w}_{i}[k]+\frac{2 \mu y[k] r_{i}[k]}{\widehat{P}_{r_{i}}[k]+\epsilon},
$$

with $\epsilon$ being a small value to prevent zero division and where the power-estimate $\widehat{P}_{r_{i}}[k]$ of the $i^{\prime}$ th reference signal $r_{i}[k]$ can be computed by a recursive averaging:

$$
\widehat{P}_{r_{i}}[k+1]=\beta \widehat{P}_{r_{i}}[k]+(1-\beta) r_{i}^{2}[k],
$$

with $\beta$ being a smoothing parameter (lower, but close to 1 ).

The gradient search only needs to be performed in case one or both of the directional interferers are present. In case the desired speech is present during the adaptation, the gradient search will not behave robustly in practice. This nonrobust behaviour is caused by leakage of speech in the noise references $r_{1}$ and $r_{2}$ due to either variations of the desired speaker location, microphone mismatches or reverberation (multipath) effects. To avoid adaptation during desired speech, we will apply a step-size control factor in the adaptation-rule, given by

$$
\Psi[k]=\frac{\hat{P}_{r_{1}}[k]+\widehat{P}_{r_{2}}[k]}{\widehat{P}_{r_{1}}[k]+\widehat{P}_{r_{2}}[k]+\widehat{P}_{p}[k]+\epsilon},
$$

where $\hat{P}_{r_{1}}[k]+\hat{P}_{r_{2}}[k]$ is an estimate of the noise power and $\hat{P}_{p}[k]$ is an estimate of the primary signal $p[k]$ that contains mainly desired speech. The power estimate $\hat{P}_{p}[k]$ is, just as for the reference-signal powers $\hat{P}_{r_{1}}$ and $\hat{P}_{r_{2}}$, obtained via recursive averaging:

$$
\hat{P}_{p}[k+1]=\beta \hat{P}_{p}[k]+(1-\beta) p^{2}[k] .
$$

We can see that the value of $\Psi[k]$ will be small when the desired speech is dominating, while $\Psi[k]$ will be much larger (but lower than 1) when either the directional interferers or spherically isotropic noise is dominating. As it is beneficial to have a low amount of noise components in the power estimate $\hat{P}_{p}[k]$, we found that $\alpha=0.25$ is a good choice. 


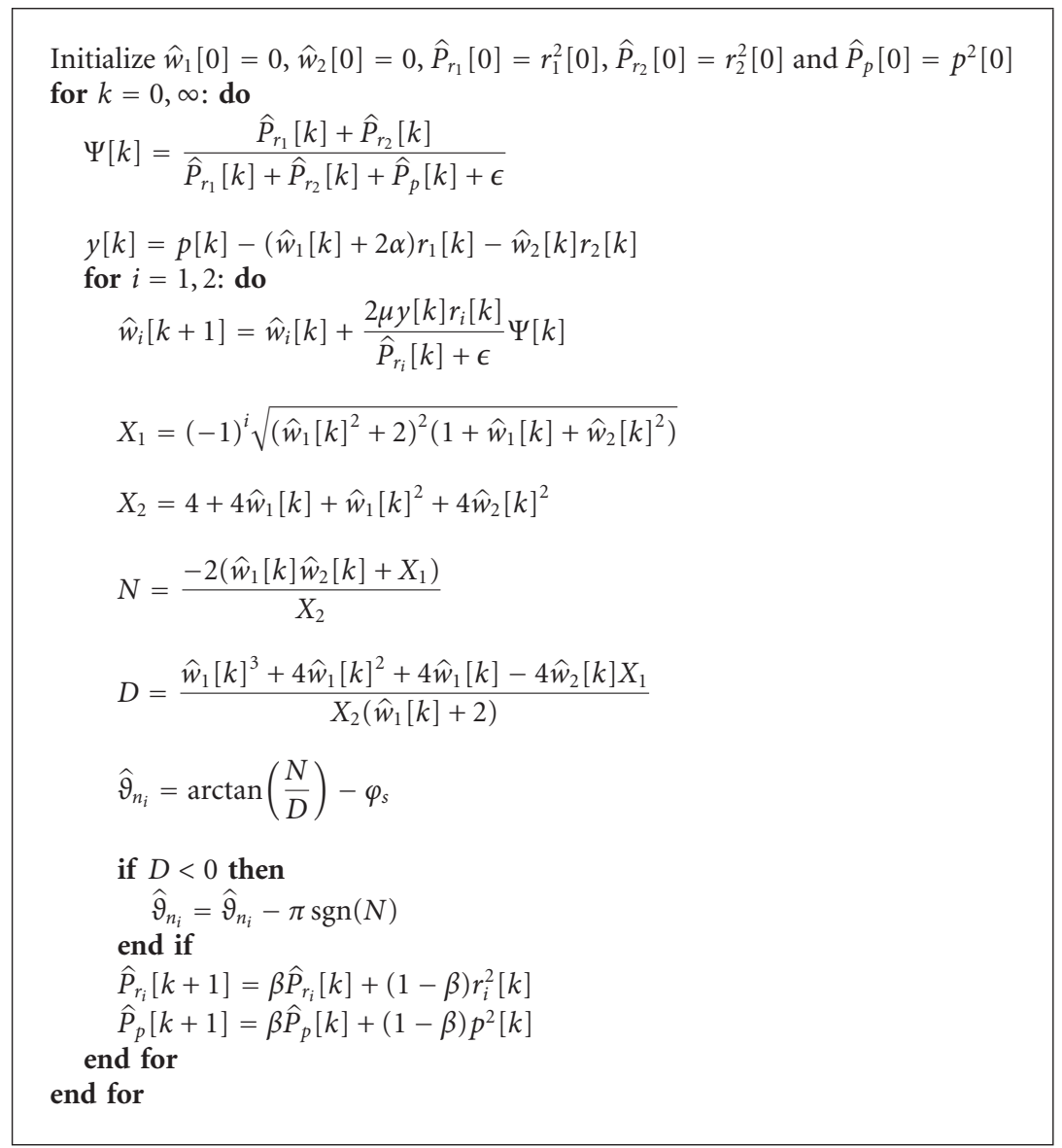

Algorithm 1: Optimal null-steering for two directional interferers.

The algorithm now looks as shown in Algorithm 1.

As can be seen in the algorithm, the two weights $\widehat{w}_{1}[k]$ and $\widehat{w}_{2}[k]$ are adapted based on a gradient-search method. Based on these two weights, a computation with arctanfunction is performed to obtain the angles of the directional interferers $\widehat{\vartheta}_{n_{i}}$ with $i=1,2$.

\section{Validation}

6.1. Directivity Pattern for Directional Interferers. First, we show the beampatterns for a number of situations where two nulls are placed. In Table 1, we show the computed values for the direct pattern synthesis for 4 different situations, where nulls are placed at different angles. Furthermore, we assume that there is no isotropic noise present.

As was explained in Section 3.1, we can obtain two different sets of solutions for $\tilde{\varphi}_{s}, \tilde{\alpha}$, and $\widetilde{S}$. In Table 1 , we show the set of solutions where $\tilde{\alpha}$ is positive.

Similarly, in Table 2, we show the computed values for $\tilde{w}_{1}$ and $w_{2}$ in the GSC structure as explained in Section 4 for the same situations as for the direct pattern synthesis.

The polar-plots resulting from the computed values in Tables 1 and 2 are shown in Figure 8. It is noted that the two examples of Section 5.1 where we analyzed the cost-function are depicted in Figures 8(b) and 8(d).
TABle 1: Computed values of $\tilde{\varphi}_{s}, \tilde{\alpha}$, and $\widetilde{S}$ for placing two nulls at $\varphi_{n_{1}}$ and $\varphi_{n_{2}}$ and having a unity response at $\varphi_{s}$.

\begin{tabular}{ccccccc}
\hline $\begin{array}{c}\varphi_{n_{1}} \\
(\mathrm{deg})\end{array}$ & $\begin{array}{c}\varphi_{n_{2}} \\
(\mathrm{deg})\end{array}$ & $\begin{array}{c}\varphi_{s} \\
(\mathrm{deg})\end{array}$ & $\begin{array}{c}\tilde{\varphi}_{s} \\
(\mathrm{deg})\end{array}$ & $\tilde{\alpha}$ & $\tilde{S}$ & $Q_{S}$ \\
\hline 45 & 180 & 90 & $\mathbf{2 9 2 . 5}$ & $\mathbf{0 . 2 7 7}$ & $\mathbf{1 . 1 4 1}$ & 0.61 \\
0 & 180 & 90 & $\mathbf{9 0}$ & $\mathbf{0}$ & $\mathbf{1 . 0}$ & 3.0 \\
0 & 225 & 90 & $\mathbf{1 1 2 . 5}$ & $\mathbf{0 . 2 7 7}$ & $\mathbf{1 . 0 5 8}$ & 3.56 \\
0 & 0 & 90 & $\mathbf{0}$ & $\mathbf{0 . 5}$ & $\mathbf{2}$ & 0.75 \\
\hline
\end{tabular}

TABLE 2: Computed values of $\tilde{w}_{1}$ and $w_{2}$ for placing two nulls at $\varphi_{n_{1}}$ and $\varphi_{n_{2}}$ and having a unity response at $\varphi_{s}$.

\begin{tabular}{cccccc}
\hline $\begin{array}{c}\varphi_{n_{1}} \\
(\mathrm{deg})\end{array}$ & $\begin{array}{c}\varphi_{n_{2}} \\
(\mathrm{deg})\end{array}$ & $\begin{array}{c}\varphi_{s} \\
(\mathrm{deg})\end{array}$ & $\widetilde{\boldsymbol{w}}_{1}$ & $\boldsymbol{w}_{2}$ & $Q_{S}$ \\
\hline 45 & 180 & 90 & $\sqrt{\mathbf{2}}$ & $-\frac{\mathbf{1}}{\mathbf{2}} \sqrt{\mathbf{2}}$ & 0.61 \\
0 & 180 & 90 & $\mathbf{0}$ & $\mathbf{0}$ & 3.0 \\
0 & 225 & 90 & $\frac{-\mathbf{2}}{\mathbf{2 + \sqrt { 2 }}}$ & $\frac{-\mathbf{1}}{\mathbf{2 + \sqrt { \mathbf { 2 } }}}$ & 3.56 \\
0 & 0 & 90 & $-\mathbf{2}$ & $-\mathbf{1}$ & 0.75 \\
\hline
\end{tabular}




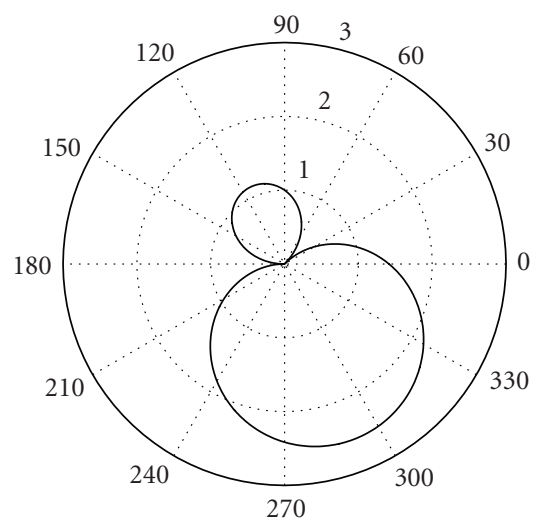

(a)

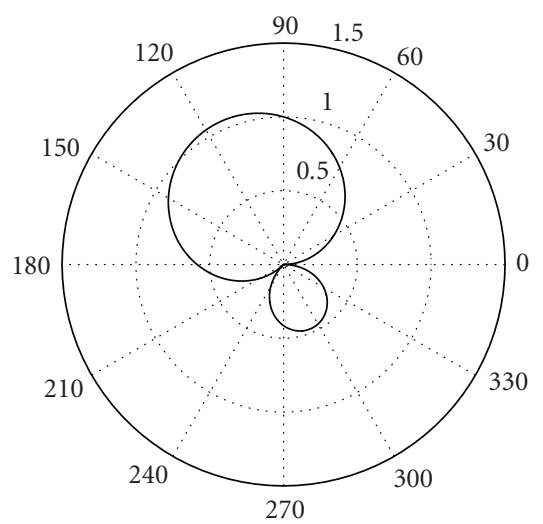

(c)

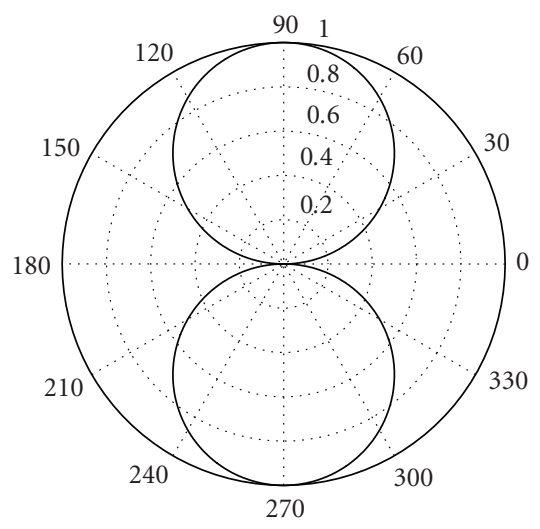

(b)

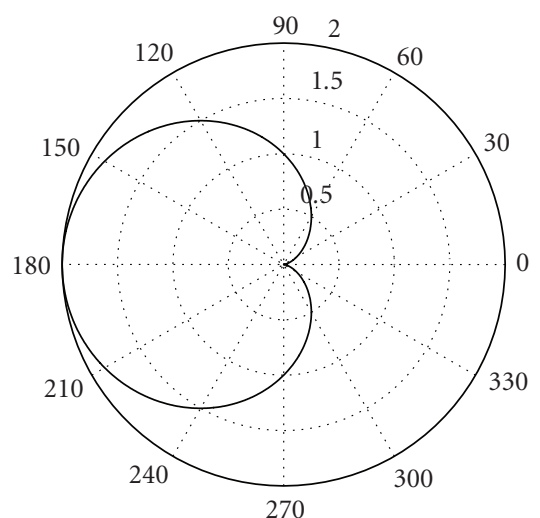

(d)

Figure 8: Azimuthal polar-plots for the placement of two nulls with nulls placed at (a) 45 and 180 degrees, (b) 0 and 180 degrees, (c) 0 and 225 degrees and (d) 0 , and 0 degrees (two identical nulls).

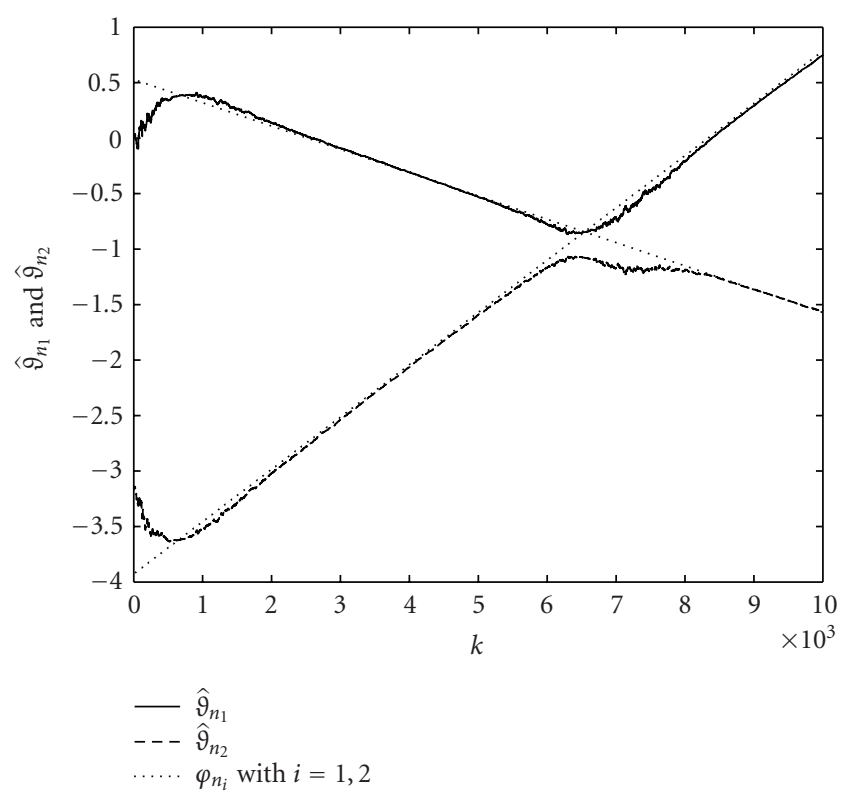

FIGURE 9: Simulation of the null-steering algorithm with two directional interferers only where $\sigma_{n_{1}}^{2}=\sigma_{n_{2}}^{2}=1$.
From the plots in Figure 8, it can be seen that if one of the two null-angles is close to the desired source angle (e.g., in Figure 8(a)), the directivity index becomes worse. Because of this poor directivity index, the null-steering method as is proposed in this paper will only be useful when either azimuthal angle of the two directional interferers is not very close to the azimuthal angle of the desired source. When we limit the main-beam to be steered maximally 90 degrees away from the desired direction, that is, $\left|\widetilde{\varphi}_{s}-\varphi_{s}\right|<\pi / 2$, we avoid a poor directivity index. For example, in Figure $8(d)$ such a situation is shown where the main-beam is steered 90 degrees away from the desired direction. In case the two directional interferers will change quickly from 0 to 180 degrees, the adaptive algorithm will automatically adapt and removes these two directional interferers at 180 degrees. As only two weights are used in the adaptive algorithm, the convergence to the optimal weights will be very fast.

6.2. Gradient Search Algorithm. Next, we validate the tracking behaviour of the gradient update algorithm, as proposed in Section 5.4. We perform a simulation, where we have a desired source at 90 degrees and where we linearly increase the angle of a first undesired directional interferer (ranging 


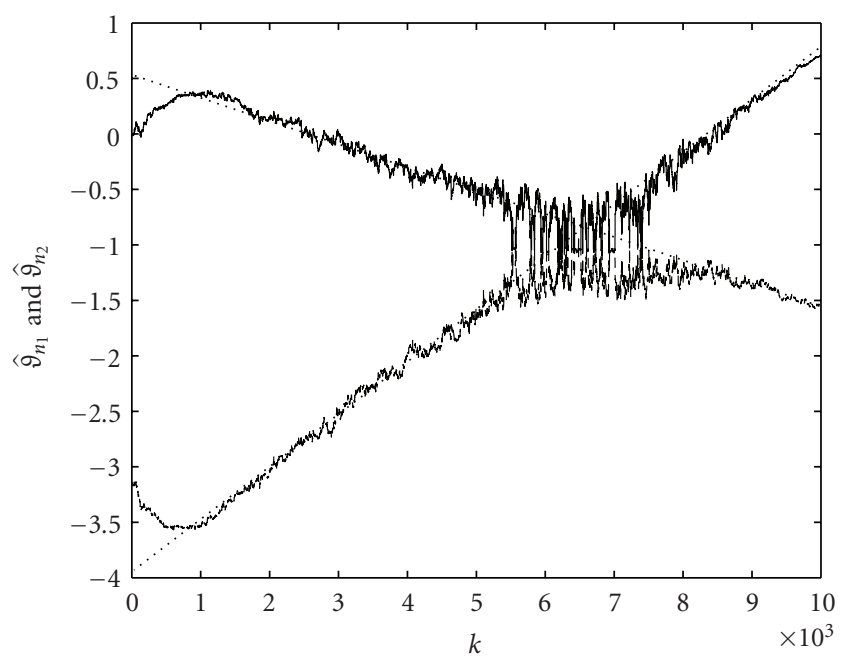

$-\hat{\vartheta}_{n_{1}}$
$---\hat{\vartheta}_{n_{2}}$
$\cdots \cdots \varphi_{n_{i}}$ with $i=1,2$

(a)

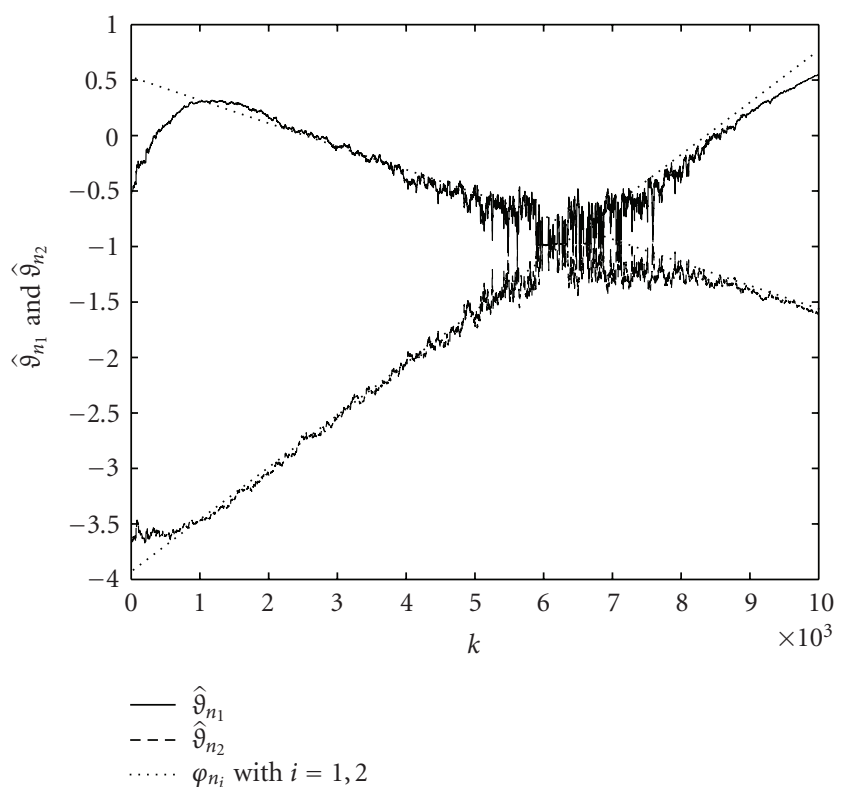

(b)

FIGURE 10: Simulation of the null-steering algorithm with two directional interferers where $\sigma_{n_{1}}^{2}=\sigma_{n_{2}}^{2}=1$ and with a desired source where $\sigma_{s}^{2}=1 / 16$ with $\varphi_{s}=90$ degrees (a) and $\varphi_{s}=60$ degrees (b).

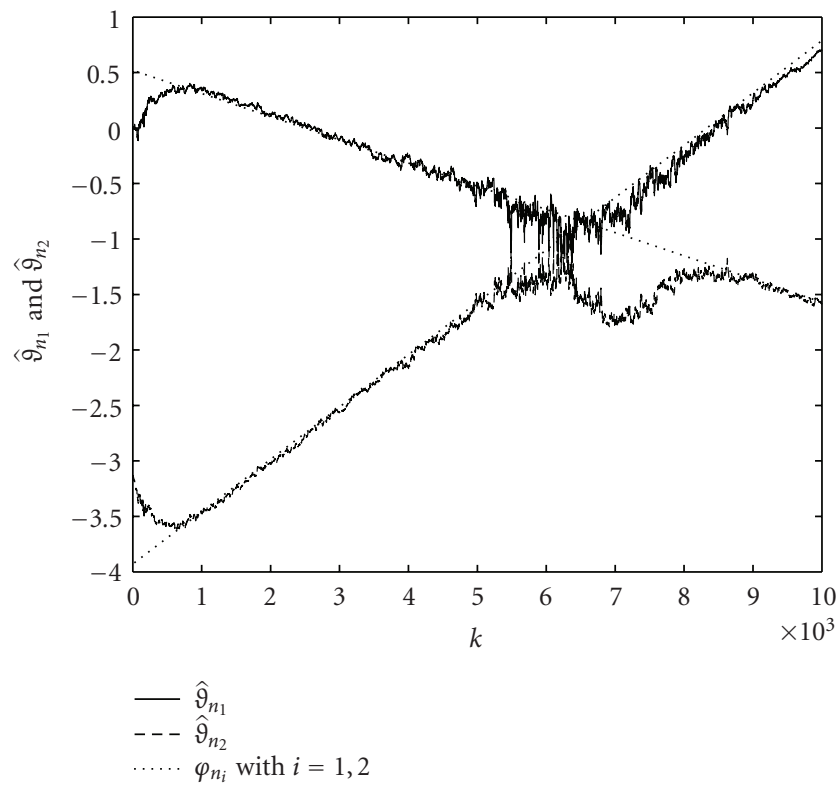

(a)

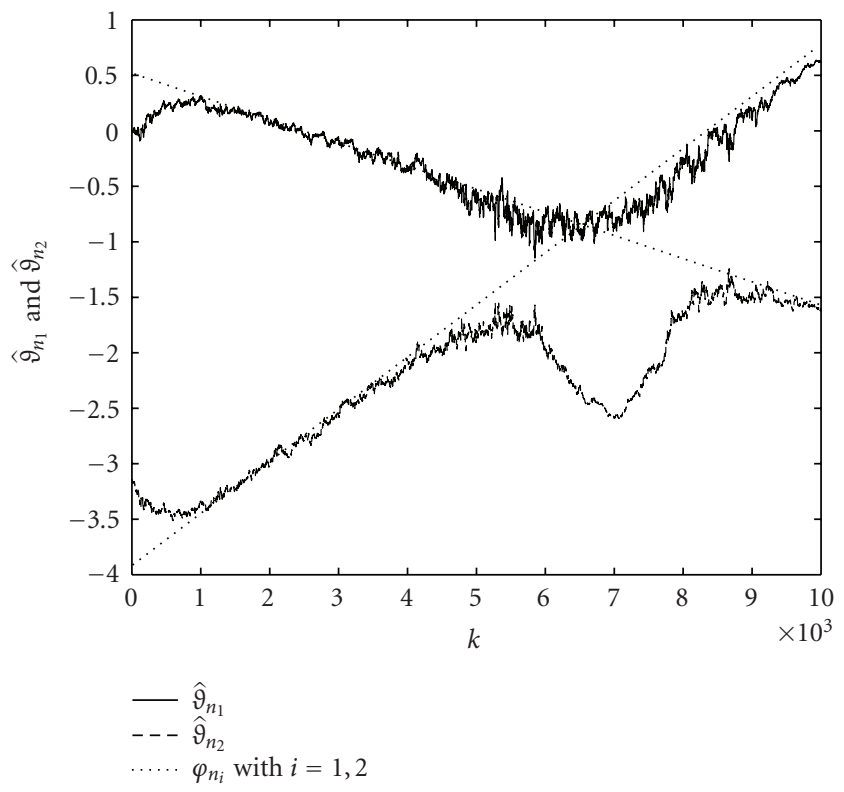

(b)

FIGURE 11: Simulation of the null-steering algorithm with two directional interferers where $\sigma_{n_{1}}^{2}=\sigma_{n_{2}}^{2}=1$ and with (spherically isotropic) spherical isotropic noise $(\gamma=1 / 3)$, where $\sigma_{d}^{2}=1 / 16(\mathrm{a})$ and $\sigma_{d}^{2}=1 / 4(\mathrm{~b})$.

from 135 to 45 degrees) and we linearly decrease the angle of a second undesired directional interferer (ranging from 30 degrees to -90 degrees) in a time-span of 10000 samples. For the simulation, we used $\alpha=0.25, \mu=0.02$, and $\beta=0.95$.

First, we simulate the situation, where only two directional interferers are present. The two directional interferers are uncorrelated white random-noise signals with variance $\sigma_{n_{i}}^{2}=1$. The results are shown in Figure 9. It can be seen that $\hat{\vartheta}_{n_{1}}$ and $\hat{\vartheta}_{n_{2}}$ do not cross (in contrast to the angles of the directional interferers $\varphi_{n_{1}}$ and $\varphi_{n_{2}}$ ). The first null placed at $\hat{\vartheta}_{n_{1}}$ adapts very well, while the second null, placed at $\widehat{\vartheta}_{n_{2}}$, is poorly adapted. The reason for this was explained in Section 5.1.

Similarly, we simulate the situation with the same two directional interferers but now together with a desired 


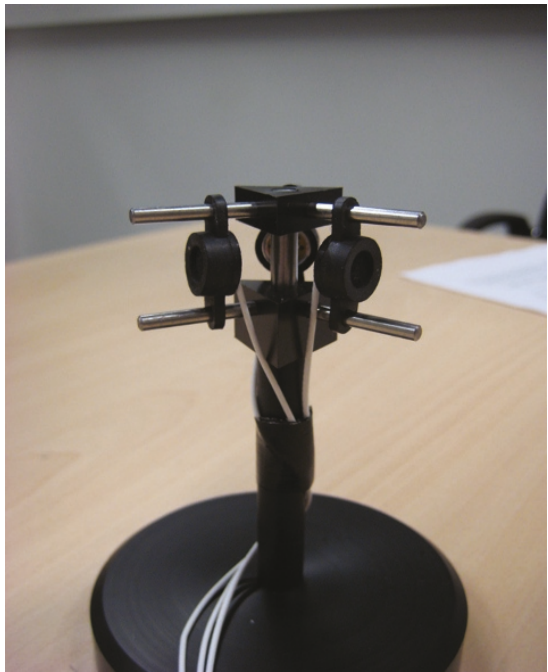

Figure 12: Microphone array with 3 outward facing cardioid microphones.

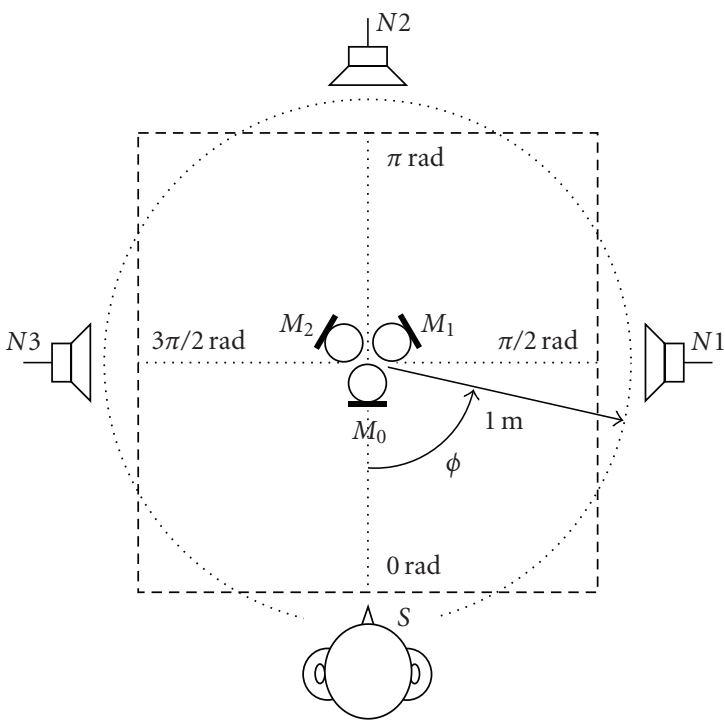

Figure 13: Practical setup of the microphone array.

source-signal $s[k]$. The desired source is modelled as a whitenoise signal, with a variance $\sigma_{s}^{2}=1 / 16$. The result is shown in Figure 10(a). We see that due to the adaptation-noise (caused by $s[k]$ ), there is more variance in the estimates of the angles $\hat{\vartheta}_{n_{1}}$ and $\hat{\vartheta}_{n_{2}}$. In contrast to the situation with two directional interferers only, we see that there is a region where $\widehat{\vartheta}_{n_{1}}=\widehat{\vartheta}_{n_{2}}$.

To show how the adaptation behaviour looks in presence of variation in the desired source location, we do a similar simulation as above, but now with $\varphi_{s}$ set to 60 degrees, while the desired source is coming from 90 degrees. This means that there will be leakage of the desired source signal into the noise reference signals $r_{1}[k]$ and $r_{2}[k]$. The results are shown in Figure 10(b). Here, it can be seen that the adaptation shows a small offset if one of the directional source angles comes close to the desired source angle. For example, at the

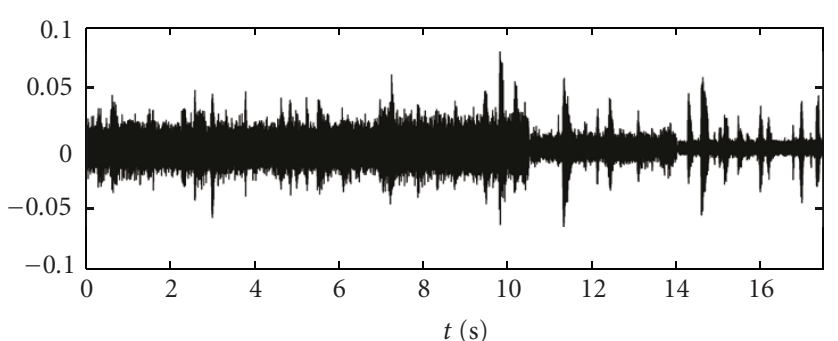

(a) Cardioid to 0 degrees, that is, $M_{0}$

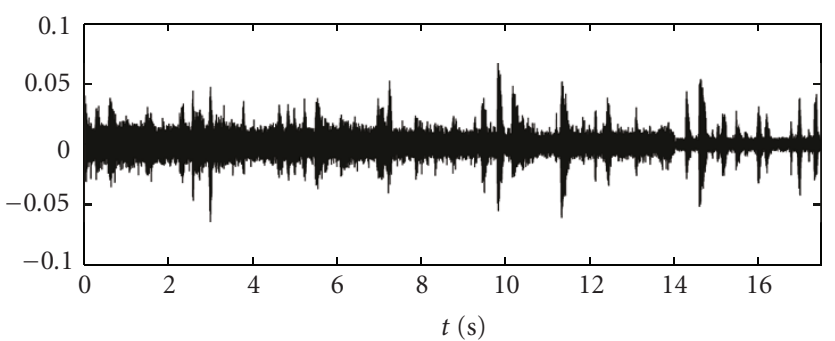

(b) Proposed adaptive null-steering algorithm

FIGURE 14: Results of the real-life experiment (waveform).

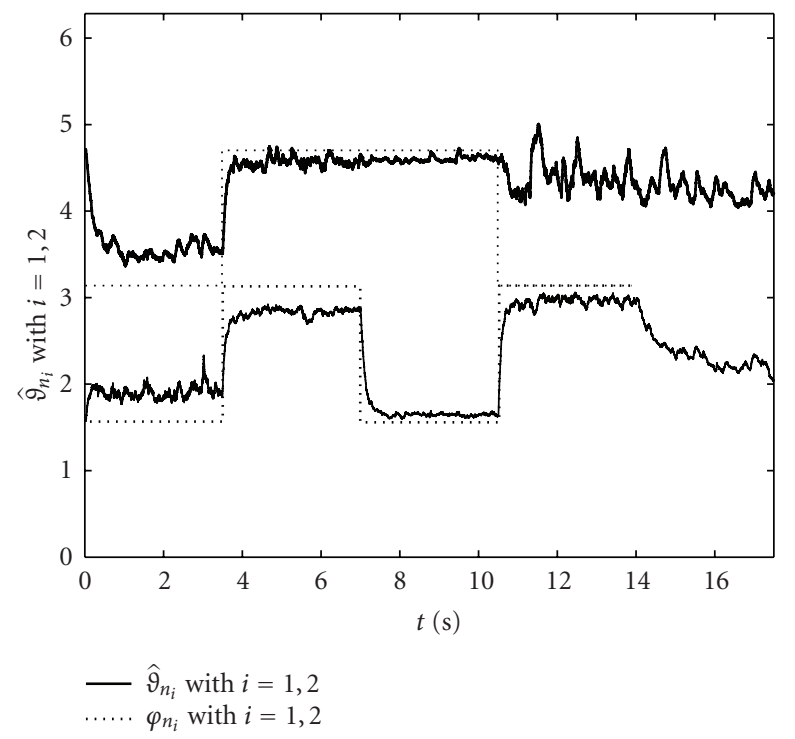

FIGURE 15: Results of the real-life experiment (angle estimates).

end of the simulation where $k=10000$, this can be clearly seen for $\hat{\vartheta}_{n_{1}}$.

Finally, we simulate the situation of the same directional interferers, but now in a spherical isotropic noise situation. As was explained in Section 5.2, isotropic noise can be modelled by adding uncorrelated additive white-noise to the three eigenbeams $E_{m}, E_{d}^{0}$, and $E_{d}^{\pi / 2}$ with variances $\sigma_{d}^{2}, \sigma_{d}^{2} \gamma$, and $\sigma_{d}^{2} \gamma$, respectively. Here $\gamma=1 / 3$ for spherically isotropic noise and $\gamma=1 / 2$ for cylindrically isotropic noise. In our simulation, we use $\gamma=1 / 3$. The results are shown in Figures 11(a) and 11(b) with variances $\sigma_{d}^{2}=1 / 16$ and $\sigma_{d}^{2}=1 / 4$, respectively. When the variance of the diffuse noise gets 


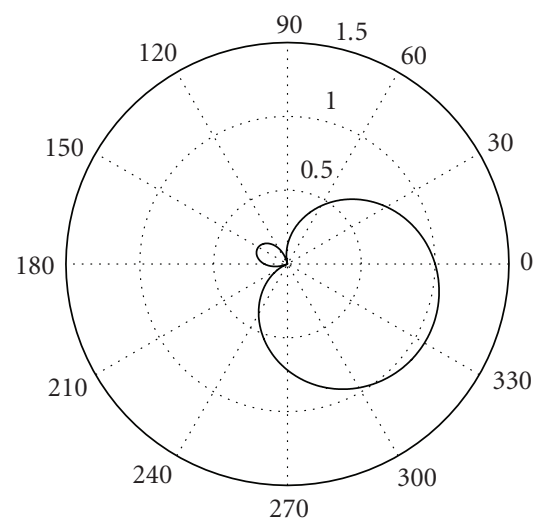

(a) $t=2.5$ seconds

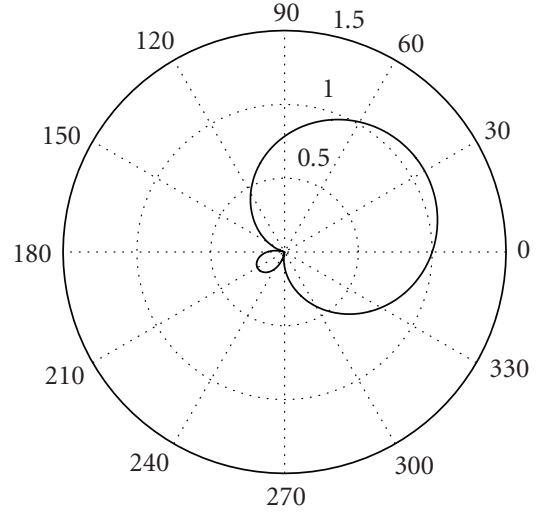

(b) $t=6$ seconds

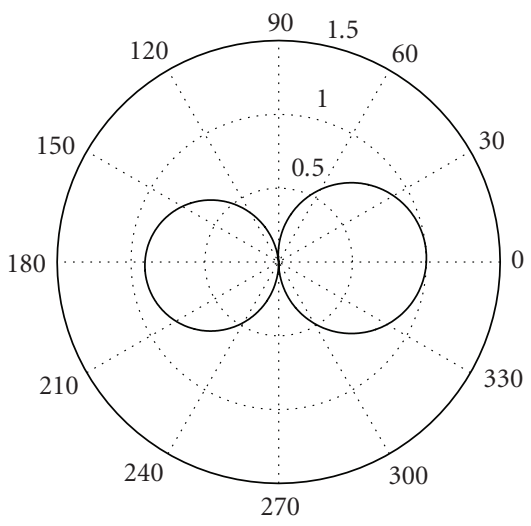

(c) $t=9.5$ seconds

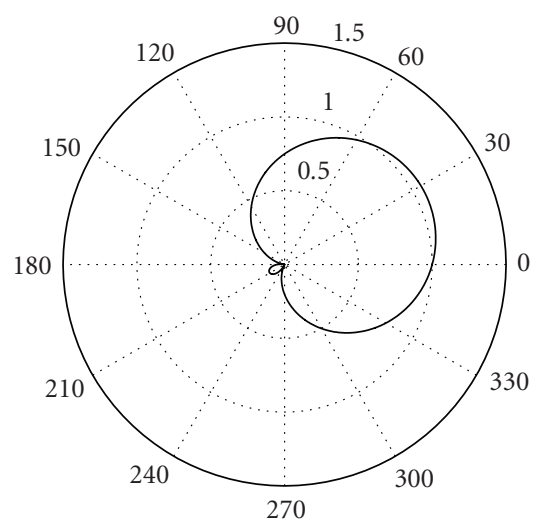

(d) $t=13$ seconds

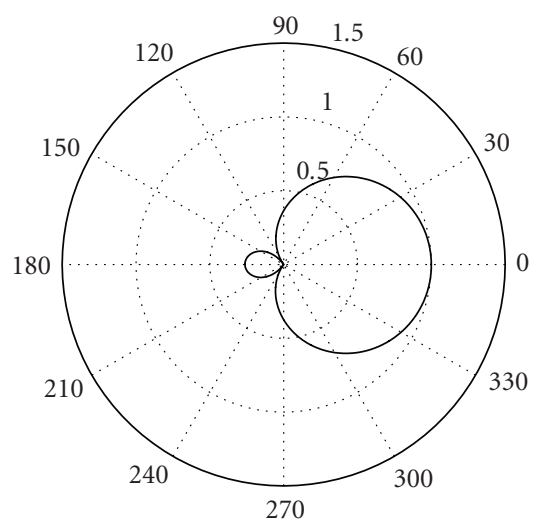

(e) $t=16.5$ seconds

FIgURE 16: Polar-plot results of the real-life experiment.

larger compared to the directional interferers, the adaptation will be influenced by the diffuse noise that is present. The larger the diffuse noise, the more the final beampattern will resemble the hypercardioid. If diffuse noise would be dominant over the directional interferers, the estimates $\varphi_{n_{1}}$ and $\varphi_{n_{2}}$ will be equal to $90-109$ degrees, and $90+109$ degrees, respectively, (or -0.33 and -2.81 radians, resp.).

6.3. Real-Life Experiments. To validate the null-steering algorithm in real-life, we used a microphone array with 3 outward facing cardioid electret microphones, as shown in Figure 12. As directional cardioid microphones have openings on both sides, the microphones are placed in rubber holders, enabling sound to enter both sides of the directional microphones.

The type of microphone elements used for this array is the Primo EM164 cardioid microphones [17]. These elements are placed uniformly on a circle with a radius of $1 \mathrm{~cm}$. This radius is sufficient for the construction of eigenbeams up to a frequency of $4 \mathrm{KHz}$.

For the experiment, we placed the array on a table in a moderately reverberant room (conferencing-room) with a $T_{60}$ of approximately 200 milliseconds. As shown in the setup in Figure 13, all directional sources are placed at a distance of 1 meter from the array (at discrete azimuthal angles: $\phi=0, \pi / 2, \pi$, and $3 \pi / 2$ radians), while diffuse noise was generated via four loudspeakers, placed close to the walls and each facing diffusers hanging on the walls. The level of the diffuse noise is $12 \mathrm{~dB}$ lower compared to the directional (interfering) sources. The experiment is done in a time-span of 17.5 seconds, where we switch the directional sources as shown in Table 3.

We use mutually uncorrelated white random-noise sequences for the directional sources $N 1, N 2$, and $N 3$ played by loudspeakers and use speech for the desired sound-source S.

For the algorithm, we use discrete-time signals with a sample-rate of $8 \mathrm{KHz}$. Furthermore, we used $\alpha=0.25, \mu=$ 0.001 , and $\beta=0.95$.

Figure 14(a) shows the waveform obtained from microphone \#0 $\left(M_{0}\right)$, which is a cardioid pointed with its mainlobe to 0 radians. This waveform is compared with the resulting waveform of the null-steering algorithm, and is shown in Figure 14(b). As the proposed null-steering algorithm is able to steer nulls toward the directional interferers, the direct part of the interferers is removed effectively (this can be seen by the lower noise-level in Figure 14(b) in the time-frame from $0-10.5$ seconds). In the segment from 10.5-14 seconds (where there is only a single directional interferer at $\phi=\pi$ radians), it can be seen that the null-steering algorithm is able to reject this interferer just as good as the single cardioid microphone. 
TABLE 3: Switching of sound-sources during the real-life experiment.

\begin{tabular}{|c|c|c|c|c|c|c|}
\hline Source & angle $\phi(\mathrm{rad})$ & $0-3.5(\mathrm{~s})$ & $3.5-7(\mathrm{~s})$ & $7-10.5(\mathrm{~s})$ & $10.5-14(\mathrm{~s})$ & $14 \mathrm{~s}-17.5(\mathrm{~s})$ \\
\hline N1 & $\pi / 2$ & active & - & active & - & - \\
\hline N2 & $\pi$ & active & active & - & active & - \\
\hline N3 & $3 \pi / 2$ & - & active & active & - & - \\
\hline$S$ & 0 & active & active & active & active & active \\
\hline
\end{tabular}

In Figure 15, the resulting angle-estimates from the nullsteering algorithm are shown. Here, it can be seen that the angle-estimation for the first three segments of 3.5 seconds is done accurately. For the fourth segment, there is only a single point interferer. In this segment, only a single angle-estimation is stable, while the other angle-estimation is highly influenced by the diffuse noise. Finally, in the fifth segment, only diffuse noise is present and the final beampattern will optimize the directivity-index, leading to a more hypercardioid beampattern steered with its main-lobe to 0 degrees (as explained in Section 6.2).

Finally, in Figure 16, the resulting polar-patterns from the null-steering algorithm are shown for some discrete time-stamps. Again, it becomes clear that the null-steering algorithm is able to steer the nulls toward the angles where the interferers are coming from.

\section{Conclusions}

We analyzed the construction of a first-order superdirectional response in order to obtain a unity response for a desired azimuthal angle and to obtain a placement of two nulls to undesired azimuthal angles to suppress two directional interferers. We derived a gradient search algorithm to adapt two weights in a generalized sidelobe canceller scheme. Furthermore, we analyzed the cost-function of this gradient search algorithm, which was found to be convex. Hence a global minimum is obtained in all cases. From the two weights in the algorithm and using a four-quadrant inversetangent operation, it is possible to obtain estimates of the azimuthal angles where the two directional interferers are coming from. Simulations and real-life experiments show a good performance in moderate reverberant situations.

\section{Appendix}

\section{Proofs}

Maximum Directivity Factor $Q_{S}$. We prove that for

$$
Q_{S}\left(\varphi_{1}, \varphi_{2}\right)=\frac{6\left(1-\cos \varphi_{1}\right)\left(1-\cos \varphi_{2}\right)}{5+3 \cos \left(\varphi_{1}-\varphi_{2}\right)}
$$

with $\varphi_{1}, \varphi_{2} \in[0,2 \pi]$, a maximum $Q_{S}=4$ is obtained for $\varphi_{1}=\arccos (-1 / 3)$ and $\varphi_{2}=2 \pi-\arccos (-1 / 3)$.
Proof. First, we compute the numerator of the partial derivative $\partial Q_{S} / \partial \varphi_{1}$ and set this derivative to zero:

$$
\begin{aligned}
& 6\left(1-\cos \varphi_{1}\right) \sin \varphi_{1}\left[5+3 \cos \left(\varphi_{1}-\varphi_{2}\right)\right] \\
& \quad+6\left(1-\cos \varphi_{1}\right)\left(1-\cos \varphi_{2}\right) 3 \sin \left(\varphi_{1}-\varphi_{2}\right)=0 .
\end{aligned}
$$

The common factor $6\left(1-\cos \varphi_{1}\right)$ can be removed, resulting in

$\sin \varphi_{1}\left(5+3 \cos \left(\varphi_{1}-\varphi_{2}\right)\right)+3\left(1-\cos \varphi_{1}\right) \sin \left(\varphi_{1}-\varphi_{2}\right)=0$.

Similarly, setting the partial derivative $\partial Q_{S} / \partial \varphi_{2}$ equal to zero, we get

$\sin \varphi_{2}\left(5+3 \cos \left(\varphi_{2}-\varphi_{1}\right)\right)+3\left(1-\cos \varphi_{2}\right) \sin \left(\varphi_{2}-\varphi_{1}\right)=0$.

Combining (A.3) and (A.4) gives

$$
\begin{aligned}
\frac{\sin \varphi_{1}}{1-\cos \varphi_{1}} & =\frac{-3 \sin \left(\varphi_{1}-\varphi_{2}\right)}{5+3 \cos \left(\varphi_{1}-\varphi_{2}\right)} \\
& =\frac{3 \sin \left(\varphi_{2}-\varphi_{1}\right)}{5+3 \cos \left(\varphi_{2}-\varphi_{1}\right)}=\frac{-\sin \varphi_{2}}{1-\cos \varphi_{2}},
\end{aligned}
$$

or alternatively

$$
\frac{2 \sin \left(\varphi_{1} / 2\right) \cos \left(\varphi_{1} / 2\right)}{2 \sin ^{2}\left(\varphi_{1} / 2\right)}=\cot \left(\frac{\varphi_{1}}{2}\right)=-\cot \left(\frac{\varphi_{2}}{2}\right),
$$

with $\varphi_{1}, \varphi_{2} \in[0, \pi]$.

From (A.6), we can see that $\varphi_{1} / 2+\varphi_{2} / 2=\pi\left(\right.$ or $\varphi_{1}+\varphi_{2}=$ $2 \pi$ ) and can derive

$$
\begin{gathered}
\cos \varphi_{2}=\cos \left(2 \pi-\varphi_{1}\right)=\cos \varphi_{1}, \\
\sin \varphi_{2}=\sin \left(2 \pi-\varphi_{1}\right)=-\sin \varphi_{1} .
\end{gathered}
$$

Using (A.7) and (A.8) in (A.1) gives

$$
Q_{S}=\frac{6\left(1-\cos \varphi_{1}\right)^{2}}{5+3\left(2 \cos \varphi_{1}-1\right)}=\frac{6\left(1-\cos \varphi_{1}\right)^{2}}{2+6 \cos ^{2} \varphi_{1}}=\frac{6(1-x)^{2}}{2+6 x^{2}}
$$

with $x=\cos \varphi_{1} \in[-1,1]$.

We can compute the optimal value for $x$ by differentiation of (A.9) and setting the result to zero:

$$
\begin{aligned}
& -12(1-x)\left(2+6 x^{2}\right)-6(1-x)^{2} 12 x=0 \\
& \equiv-2-6 x^{2}-6 x+6 x^{2}=0 .
\end{aligned}
$$

Solving (A.10) gives $x=\cos \varphi_{1}=-1 / 3$ and consequently, $\varphi_{1}=\arccos (-1 / 3)$ and $\varphi_{2}=2 \pi-\arccos (-1 / 3)$. Via (A.9), we can see that for these values, we have $Q_{S}=4$. 


\section{Acknowledgment}

The author likes to thank Dr. A. J. E. M. Janssen for his valuable suggestions.

\section{References}

[1] G. W. Elko, F. Pardo, D. Lopez, D. Bishop, and P. Gammel, "Surface-micromachined mems microphone," in Proceedings of the 115th AES Convention, p. 1-8, October 2003.

[2] P. L. Chu, "Superdirective microphone array for a set-top video conferencing system," in Proceedings of the IEEE International Conference on Acoustics, Speech, and Signal Processing (ICASSP '97), vol. 1, pp. 235-238, Munich, Germany, April 1997.

[3] R. L. Pritchard, "Maximum directivity index of a linear point array," Journal of the Acoustical Society of America, vol. 26, no. 6, pp. 1034-1039, 1954.

[4] H. Cox, "Super-directivity revisited," in Proceedings of the 21st IEEE Instrumentation and Measurement Technology Conference (IMTC '04), vol. 2, pp. 877-880, May 2004.

[5] G. W. Elko and A. T. Nguyen Pong, "A simple first-order differential microphone," in Proceedings of the IEEE Workshop on Applications of Signal Processing to Audio and Acoustics (WASPAA '95), pp. 169-172, New Paltz, NY, USA, October 1995.

[6] G. W. Elko and A. T. Nguyen Pong, "A steerable and variable first-order differential microphone array," in Proceedings of the IEEE International Conference on Acoustics, Speech, and Signal Processing (ICASSP '97), vol. 1, pp. 223-226, Munich, Germany, April 1997.

[7] M. A. Poletti, "Unified theory of horizontal holographic sound systems," Journal of the Audio Engineering Society, vol. 48, no. 12, pp. 1155-1182, 2000.

[8] H. Cox, R. M. Zeskind, and M. M. Owen, "Robust adaptive beamforming," IEEE Transactions on Acoustics, Speech, and Signal Processing, vol. 35, no. 10, pp. 1365-1376, 1987.

[9] R. M. M. Derkx and K. Janse, "Theoretical analysis of a firstorder azimuth-steerable superdirective microphone array," IEEE Transactions on Audio, Speech and Language Processing, vol. 17, no. 1, pp. 150-162, 2009.

[10] Y. Huang and J. Benesty, Audio Signal Processing for Next Generation Multimedia Communication Systems, Kluwer Academic Publishers, Dordrecht, The Netherlands, 1st edition, 2004.

[11] H. Teutsch, Modal Array Signal Processing: Principles and Applications of Acoustic Wavefield Decomposition, Springer, Berlin, Germany, 1st edition, 2007.

[12] L. J. Griffiths and C. W. Jim, "An alternative approach to linearly constrained adaptive beamforming," IEEE Transactions on Antennas and Propagation, vol. 30, no. 1, pp. 27-34, 1982.

[13] R. M. M. Derkx, "Optimal azimuthal steering of a firstorder superdirectional microphone response," in Proceedings of the 11th International Workshop on Acoustic Echo and Noise Control (IWAENC '08), Seattle, Wash, USA, September 2008.

[14] J.-H. Lee and Y.-H. Lee, "Two-dimensional adaptive array beamforming with multiple beam constraints using a generalized sidelobe canceller," IEEE Transactions on Signal Processing, vol. 53, no. 9, pp. 3517-3529, 2005.

[15] W. Kaplan, Maxima and Minima with Applications: Practical Optimization and Duality, John Wiley \& Sons, New York, NY, USA, 1999.

[16] B. H. Maranda, "The statistical accuracy of an arctangent bearing estimator," in Proceedings of the Oceans Conference
(OCEANS '03), vol. 4, pp. 2127-2132, San Diego, Calif, USA, September 2003.

[17] R. M. M. Derkx, "Spatial harmonic analysis of unidirectional microphones for use in superdirective beamformers," in Proceedings of the 36th International Conference: Automotive Audio, Dearborn, Mich, USA, June 2009. 